\title{
Estado actual de las investigaciones sobre líquenes foliícolas en la región Neotrópica, con un análisis biogeográfico preliminar
}

\author{
Robert Lücking \\ Abteilung Spezielle Botanik (Biologie V), Universität Ulm, D-89069 Ulm, Germany
}

\begin{abstract}
An overview over the present state of investigations on foliicolous lichens in the Neotropics is provided. Historically, a division can be made into (1) the initial period, dominated by the work of J. Müller Argoviensis, (2) the monography of R. Santesson, (3) the Brazilian phase, characterized by the Brazilian mycologist A. C. Batista and his working group, and (4) the modern period, with detailed monographical and floristical studies and an increasing interest in the ecology of foliicolous lichens and their use as bioindicators. We now have rather good knowledge of the taxonomy and systematics of most groups, although new species are continuously described and some families and genera are in need of revision. Detailed floristical information is available from a few regions only, and biogeographical data are therefore incomplete and controversial. Modern ecological studies have been made to some extent, yet the results and their implications have to be verified by extended studies inside and outside the Neotropics. Preliminary data indicate that foliicolous lichens are useful as bioindicators in various respects. Biogeographically, the Neotropics are characterized by a high number of exclusive species and by their affinity with tropical Africa. Within the Neotropics, biogeographical subdivisions, as established by higher plant distribution patterns, are only in a few instances supported by the distribution of foliicolous lichens. In general, subtile biogeographical conclusions with respect to foliicolous lichens are difficult, because of the wide distribution of many species and the insufficient floristic knowledge of particular regions.
\end{abstract}

Resumen: Se presenta un resumen del estado actual de investigaciones sobre líquenes foliícolas en la región Neotrópica. Historicamente, se puede hacer una división en (1) el período inicial, dominado por las obras de J. Müller Argoviensis, (2) la monografía de R. Santesson, (3) la fase brasileña, caracterizada por el micólogo Brasileño A. C. Batista y sus colaboradores, y (4) el período moderno, con estudios detallados monográficos y florísticos y un creciente interés en la ecología de líquenes foliícolas. Actualmente tenemos un conocimiento -taxonómico y sistemático relativamente bueno en la mayoría de los grupos, aunque nuevas espécies son descritas continuamente y algunas familias y géneros 
necesitan revisiones. Información florística detallada existe solamente de pocas regiones, y por esa razon los datos biogeográficos quedan incompletos y controversos. Estudios ecológicos modernos fueron realizados en varias ocasiones, pero falta verificar los resultados por estudios extensivas en otras regiones dentro y fuera del Neotrópico. Investigaciones preliminares demuestran que los líquenes foliícolas pueden ser usados como bioindicadores en diferentes formas. Biogeograficamente, la región Neotrópica esta caracterizada por un alto número de espécies exclusivos y por su afinidad con África tropical. Debido a la amplia distribución de muchos taxa y al déficit del conocimiento florístico, las subdivisiones biogeográficas dentro del Neotrópico, establecidas con patrones de distribución de plantas superiores, solo en pocos casos son apoyadas por la distribución de líquenes foliícolas.

\section{Introducción}

Un fenomeno particular de los bosques tropicales lluviosos es el crecimiento de líquenes y briófitas sobre hojas vivas de plantas vasculares (Santesson 1952; Richards 1984; Sipman \& Harris 1989; Gradstein \& Pócs 1989). Desde los primeros estudios de Fries (1823, 1830) y Fée (1824), existe una gran tradición de recolecta y investigación científica de líquenes foliícolas el Neotrópico, documentado por los trabajos de Müller Argoviensis(1885-1895), Santesson(1952), Vezda (1984), Kalb \& Vezda (1988a, b, 1990), Sipman (1990a, 1991a, b, 1992), Aptroot \& Sipman(1993a) y Lücking (1991-1995), entre otros. Alrededor de $60 \%$ de las especies aceptadas en la monografía de Santesson (1952) tienen su localidad tipo en el Neotrópico, la gran mayoría de ellas proveniendo de Brasil. Hoy dia el número de especies se conoce más que el doble de especies (Farkas \& Sipman 1993), pero el papel importante de la región Neotrópica no ha cambiado.

Otro particular es que la mayoría de estudios modernos ecológicos han sido hechos en el Neotrópico, especialmente por el grupo de S. Winkler y sus colaboradores (Nowak \& Winkler 1970-1975; Lücking 1992b-c, 1994,1995b-c;Barillas et al. 1993). También varios tratamientos ecogeográficos fueron realizados basandose en material Neotropical (Schell \& Winkler 1980; Kalb \& Vezda 1988a, 1990; Lücking \& Lücking 1995). En el presente trabajo, después de un esbozo histórico, se da una vista general sobre el estado actual del conocimiento de los líquenes foliícolas en la región Neotrópica, acompañada por una perspectiva de futuros proyectos. Lo escrito se dirige en particular a los liquenólogos radicados en América Central y Suramérica que quieren dedicarse al estudio de los líquenes foliícolas. También se intenta un análisis biogeográfico preliminar, presentando los elementos característicos para la región Neotrópica y sus subregiones.

\section{Breve historia del estudio de líquenes foliícolas Neotropicales}

La historia de estudios sobre líquenes foliícolas Neotropicales puede ser dividida en cuatro fases: (1) el período inicial (1823-1937), con las obras de J. Müller Argoviensis y otros autores, (2) la monografía de R. Santesson(1939-1952), (3) la fase Brasileña (1960-1975), dominado por el micólogo brasileño A. C. Batista y sus colaboradores, y (4) el período moderno (desde 1970), con investigaciones detalladas monográficas, floristicas y ecológicas.

El período inicial (1823-1937). El período inicial comienza con los primeros tratamientos de líquenes foliícolas hechos por Fries $(1823,1830)$ y Fée (1824). La mayoría de los taxa descritos por ellos provinieron de las Antillas, documentando así el papel que hasta hoy día juega el Neotrópico en los estudios de estos organismos. Una especie común que se remonta a estos tiempos y lleva el nombre de las Antillas es Strigula antillarum (Fée) Müll. Arg. Entre 1822 y 1849 , las primeras colecciones extensas de líquenes foliícolas fueron llevadas a Europa, el centro de liquenología durante este período, y tratadas en detalle por Montagne (18381856). También tenían su orígen en el Neotrópico, coleccionadas por R. de la Sagra en Cuba y F. R. 


\begin{tabular}{|c|c|c|c|c|c|}
\hline 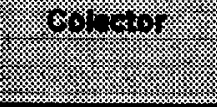 & 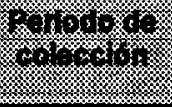 & 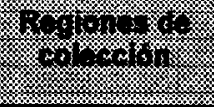 & $\left(\frac{1}{10}\right.$ & 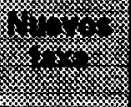 & (x) \\
\hline R. de la Sagra & $1822-1836$ & Cuba & Montagne & 1 & PC \\
\hline F.R. Leprieur & $1835-1849$ & Gulanas & Montagne & 2 & PC \\
\hline R. Spruce & $1849-1864$ & Brasil & Maller Arg., Leighton & 5 & $K(\rightarrow B M), G$ \\
\hline J.W. H. Trail & 1874 & Brasil & Stirton, Maller Arg. & 14 & $\begin{array}{l}\text { GLAM, } \\
K(\rightarrow \text { BM })\end{array}$ \\
\hline J. Puiggari & $1877-1882$ & Brasil & Muller Arg. & 34 & $\mathbf{G}$ \\
\hline c. Wright & - & Cuba & $\begin{array}{l}\text { Maller Arg., } \\
\text { Tuckerman }\end{array}$ & 5 & G, UPS, FH \\
\hline B. Balansa & $1878-1884$ & Paraguay & Maller Arg. & 2 & $\mathbf{G}$ \\
\hline A. Glaziou & 1889-1892 & Brasil & Moller Arg. & 13 & G, PC, UPS \\
\hline $\begin{array}{l}\text { H. Pittior \& } \\
\text { A. Tonduz }\end{array}$ & $1891-1892$ & Costa Rica & Maller Arg. & 6 & $\mathbf{G}$ \\
\hline W. R. Elllott & 1892 & Antilas & Vainio & 4 & TUR \\
\hline E. Ule & $1883-1903$ & Brasil & Rehm, Maller Arg. & 8 & S. G \\
\hline A. Ernst & - & Venezuela & Moller Arg. & 2 & G \\
\hline G. O. Malme & $1892-1902$ & $\begin{array}{l}\text { Brasil, } \\
\text { Paraguay }\end{array}$ & Malme, Santesson & 8 & 8 \\
\hline R. Thaxter & $1905-1913$ & Trnidad, Chlle & Valnio, Santesson & 3 & TUR \\
\hline P. C. Standley & $1927-1947$ & $\begin{array}{l}\text { America } \\
\text { Central }\end{array}$ & Santesson & 一 & F, UPS \\
\hline E. Asplund & $1939-1940$ & Ecuador & Santesson & 3 & S, UPS \\
\hline
\end{tabular}

Cuadro 1. Principales recolectores de líquenes foliícolas en el Neotrópico antes de la monografía de Santesson (1952) y los taxónomos más importantes que investigaron el material (según Santesson 1952; Hertel \& Schreiber 1988; Alava 1993). Las colecciones de líquenes de Kew (K) ahora están en el British Museum of Natural History en Londres (BM).

Leprieur en Guiana Francesa (Cuadro 1).

Más adelante, material de la región Neotrópica fue tratado por Krempelhuber(1876), Stirton (1878) y Vainio (1890,1896), basandose principalmente en colecciones de E. A. Vainio, J. W. H. Trail, W. R. Elliott y R. Thaxter de Brasil y de las Antillas. Durante los años ochenta y noventa, Müller Argoviensis (1885-1895) describió alrededor de 75 especies con material recolectado por J. Puiggari, A. F. M. Glaziou, E. Ule y R. Spruce en Brasil, por A. Ernst en Venezuela, por B. B. Balansa en Paraguay, por H. Pittiery A. Tonduz en Costa Rica y por C. Wright en Cuba. Continuaron Zahlbruckner(1908-1930),Schilling(1927)yMalme
(1924-1937), trabajando con colecciones de Brasil y Paraguay, entre otros (Cuadro 1). Aunque, con respeto a los líquenes foliícolas en general, Vainio es reconocido como el autor más importante antes de la monografía de Santesson, y Zahlbruckner sin duda alguna ha marcado la sistemática por mucho tiempo, Müller Argoviensis claramente dominaba el campo en la región Neotrópica (Cuadro 1).

Hay que mencionar que muchas especies de líquenes foliícolas originalmente fueron descritos como hongos no liquenizados, por ejemplo en los géneros Asterothyrium y Psorotheciopsis (Santesson 1952), lo que significa que todavía prodrían existir nombres viejos para líquenes 
foliícolas entre los hongos foliícolas no líquenizados, aunque la gran mayoría de estos nombres ya fue revisado por Santesson (1952).

La monografía de Santesson (1939-1952). La monografía de los líquenes foliícolas de Santesson (1952), que sin duda alguna representa un hito en la historia del estudio de estos organismos, está relacionada particularmente con la región Neotrópica. El inicio de este trabajo fueron los viajes del autor a Suramérica en los años 19391941. Durante estos viajes, los cuales le llevaron al sur de Argentina, Santesson tocó también la selva tropical de Brasil y Venezuela y recolectó material de líquenes foliícolas. Los intentos de identificar sus colecciones finalmente le dejaron reconocer que tenía que estudiar estos líquenes al nivel mundial.

Aún hoy dia la monografía de Santesson representa una de las obras liquenológicas más importantes, también a fuera del contexto de líquenes foliícolas. Santesson fue uno de los primeros autores que trataban de aplicar un sistema natural, demostrando que la septación de las ascosporas como carácter exclusivo no es apropriada para definir géneros. Su circumscripción del género Tapellaria es discutida por Hafellner (1984), pero otros autores lo aceptan, y sin duda alguna este género representa un grupo natural, uno de primeros géneros aceptados dentro de la familia colectiva Lecideaceae en donde se observa una serie de ascosporas transversalmente septadas a muriformes. Por el hecho de que un gran número de los líquenes foliícolas tiene sus relativas más cercanas en géneros o especies no foliícolas que no fueron tratados en su monografía, Santesson no tenía la posibilidad de aplicar cambios sistemáticos fundamentales, pero expresó muchas ideas sobre relaciones entre especies de diferentes géneros, las cuales después fueron realizadas por otros autores (Sérusiaux 1986; Sérusiaux \& De Sloover 1986; Vezda 1986). Por ejemplo, especies como Catillaria bouteillei (Desm.) Zahlbr. y Bacidia subternella (Nyl.) R. Sant., o Bacidia gabrielis (Müll. Arg.) Zahlbr. y Lopadium flammeum, discutidas por Santesson (1952) de ser estrechamente relacionadas, hoy dia son unidas en géneros como Fellhanera o Loflammia, respectivamente (Vezda 1986).

Interesantemente, no salieron estudios sobre lí- quenes foliícolas inmediadamente después de la monografía de Santesson (1952). La razón principal para esto podría haber sido la falta de recolecciones nuevas. El trabajo más cercano a la monografía de Santessonfuelode Ricci \& Tomaselli (1958), que reportaron algunos líquenes foliícolas del Neotrópico.

La fase brasileña (1960-1975). Esta fase está caracterizada menos por un período temporal definido que por el hecho de que los estudios taxonómicos y sistemáticos fueron dominados por un grupo pequeño de micólogos y liquenólogos Brasileños. Aunque muchos de los taxa descritos por este grupo hoy día son considerados como sinónimos, Batista y sus colaboradores han dejado un impacto importante en el estudio de líquenes foliícolas Neotropicales. Aparte de Lidia Ferraro de Argentina, es el único caso en donde científicos radicados en el Neotrópico mismo se han dedicado en detalle al estudio de estos organismos.

Durante los años sesenta y setenta, Batista y su grupo(Batista 1961; Batista \& Bezerra 1961; Batista etal. 1961,1962; Batista \& Maia 1961-1967; Batista \& Peres 1964; Bezerra etal. 1967; Cavalcanti et al. 1971, 1972a, b) describieron una gran cantidad de géneros y especies nuevos de líquenes foliícolas, tratandose en su mayoría de estadios anamórficos. El género Caprettia lleva el nombre del micólogo venezolano Corrado Capretti (Batista \& Maia 1965b). También establecieron nuevas especies o variedades de Strigula (Batista \& Cavalcanti 1964), Echinoplaca (Batista \& Poroca 1970), Mazosia (Batista et al. 1967) y Trichothelium (Bezerra etal. 1970). Asociados con este grupo fueron H. B. P. Upadhyay y L. Xavier Filho, que describieron varios taxa, incluso la nueva familia Phragmopeltecaceae(Upadhyay 1964a, b; Xavier Filho 19731976). Por lo general, las publicaciones producidas por este grupo son poco conocidas.

El período moderno (desde 1970). Esta fase esta caracterizada por tratamientos modernos florísticos y monografías, acompañados por estudios sobre la distribución, ecología y el uso de líquenes foliícolas com bioindicadores. Nuevos datos o especies fueron publicados por Novak \& Winkler (1970-75), Osorio (1970-1992), Vareschi(1973), Hertel(1974), Sérusiaux (1976, 1984, 1985, 1995), 
Ferraro(1982-1990), Arvidsson(1986), Ferraro \& Vezda (1989),Lücking (1991, 1994a, 1995a,d-g), Kalb \& Vezda(1992), Hartmann(1996), Lücking \& Ferraro (1997) y Ferraro \& Lücking (1997), entre otros. El primer representante foliícola del género Rocellinastrum fue introducido por Henssen et al. (1983). Tratamientos florísticos más detallados son los de Vezda (1984) de Cuba, Serusiaux \& de Sloover (1986) y Ferraro (1996) de Argentina, Lücking (1992a) de Costa Rica, Sipman (19901992) de Colombia, Venezuela y las Guayanas, y Barillas \& Lücking (1992) de Guatemala. Estudios con carácter monográfico fueron realizados por Kalb \& Vezda (1988a, b, 1990) sobre Mazosia, Byssoloma y la familia Gomphillaceae, por Aptroot \& Sipman(1993a) sobre la familia Trichotheliaceae en las Guayanas, y por Lücking (1995a, 1997a-c) y Lücking y Matzer (1996) sobre las familias Arthoniaceae, Opegraphaceae, y Gomphillaceae, y los géneros Trichothelium y Fellhanera, entre otros, en Costa Rica. También se empezaron a estudiar en detalle la química de líquenes foliícolas (J. Santesson 1970; Lücking et al. 1994; Elix et al. 1992, 1995) y sus hongos parásitos y parasimbiontes (Matzer 1996).

El período moderno también está caracterizado por el estudio y la descripción detallada de estructuras reproductivas asexuales particulares de líquenes foliícolas: los campilidios de la familia Ectolechiaceae y otros grupos (Sérusiaux 1986; Vezda 1986), y los hifóforos de la familia Gomphillaceae (Vezda 1973, 1979; Vezda \& Poelt 1987). Estas estructuras no fueron reconocidas apropriadamente por Santesson (1952), quien las consideró como parasimbiontes. Batista y sus colaboradores primeramente describieron hifóforos como partes de los líquenes foliícolas en donde ocurrieron, pero no establecieron la conexión con los géneros teleomórficos respectivos. Estudios sobre la distribución y la biogeografía de líquenes foliícolas son todavía escasos, limitándose a las de Schell \& Winkler (1980), Kalb \& Vezda(1988a, 1990), Lücking (1990) y Lücking \& Lücking (1995). Estudios ecológicos modernos, parcialmente incluyendo briófitos, fueron comenzados por Nowak \& Winkler(1970-1975) y continuados por S. Winkler y sus colaboradores y otros autores (Montfoort \& Ek 1990; Barillas et al. 1993; R. Lücking 1992b-c, 1994b, 1995b-c; A. Lücking 1995; Sipman 1996).

\section{Estado actual del conocimiento sobre líquenes foliícolas Neotropicales}

El siguiente capitulo está dividio en cuatro secciones: (1) Sistemática y -taxonomía; (2)Florística y biogeografía; (3) Ecología; (4) Aplicaciones. Además de una vista general, se señalan problemas que actualmente existen en las diferentes áreas de trabajo.

Sistemática y -taxonomía. En comparación con líquenes crustosos corticolas, la mayoría de los principales grupos taxonómicos (familias, géneros) de los líquenes foliícolas Neotropicales es relativamente bien conocida. Los problemas taxonómicos existentes se pueden dividir en cuatro áreas: (1) Separación -taxonómica de grupos de especies estrechamente relacionadas; (2) Descubrimiento y descripción de nuevas especies; (3) Clarificación de nombres existentes posiblemente sinónimos de otros taxa; (4) Posición sistemática de especies o géneros provisionalmente asignados a géneros o familias inapropriados.

Grupos de especies estrechamente relacionadas ocurren especialmente en los generos Calenia, Fellhanera, Mazosia, Porina, Sporopodium, Strigula, Tricharia y Trichothelium. En Calenia, la separación correcta de $C$. graphidea Vain., $C$. depressa Müll. Arg., C.phyllogena (Müll. Arg.) R. Sant. y $C$. thelotremella Vain. se hace difícil por el hecho de que especímenes bien desarollados son raros y formas intermedias son abundantes. Lo mismo ocurre en el género Trichothelium, especialmente en los grupo de T. epiphyllum y $T$. annulatum (Karst.) R. Sant. (Lücking 1997a). Muchas veces, la variabilidad morfológica o anatómica varia regionalmente, como en los géneros Porina y Strigula (Lücking 1992a, 1996a), que forman la mayor parte de la comunidad foliícola y cuya identificación correcta es por lo tanto indispensable. Por ejemplo, en el género Fellhanera existen diferencias específicas muy finas, como es el caso con F. rhapidophylli (Rehm) Vezda s. lat., la cual puede ser dividida en varias especies con base de su excípulo, hipotecio y ascosporas (Lücking 1997b).

Nuevas especies son descritas continuamente, y en géneros como Byssoloma, Calenia, 


\begin{tabular}{|c|c|c|c|}
\hline 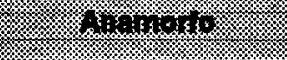 & 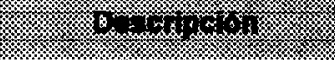 & 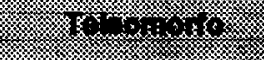 & 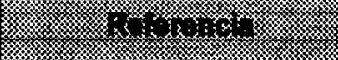 \\
\hline Aclelstomyces & Batista 1961 & Sporopodium & $\begin{array}{l}\text { Sérusiaux 1986, } \\
\text { Lucking et al. } 1997\end{array}$ \\
\hline Actinoteichus & Cavalcante et al. 1971 & Asterothyrium & Lucking ot al. 1997 \\
\hline Aderkomyces & Batista 1961 & Tricharia & Lucking ot al. 1997 \\
\hline Ameropeltomyces & Batista \& Maia 1967 & Arthonia & Lucking et al. 1997 \\
\hline Amoebomyces & Batista \& Maia 1961 & Strigula & Locking et al. 1997 \\
\hline Crocicreomyces & Batista \& Peres 1964 & Byssoloma & Lacking et al. 1997 \\
\hline Cynta & Batista \& Maia 1961 & Calopadia & Lucking et al. 1997 \\
\hline Didymaster & Batista \& Maia 1967 & Strigula & Lucking ot al. 1997 \\
\hline Didymopyonomyces & Cavalcante et al. 1972a & Dimerella & $\begin{array}{l}\text { Sórusiaux } 1992, \\
\text { Lacking et al. } 1997\end{array}$ \\
\hline Dothiomyces & Batista \& Bezerra 1961 & Byssolecania & Lucking et al. 1997 \\
\hline Killkiostroma & Batista \& Bezerra 1961 & Strigula & Lucking et al. 1997 \\
\hline Manaustrum & Cavalcante et al. $1972 a$ & Strigula & Lucking et al. 1997 \\
\hline Microxyphiomyces & Batista et al. 1961 & Tricheria, Aulexine & $\begin{array}{l}\text { Cavalcante et al. 1972a, } \\
\text { Lacking et al. } 1997\end{array}$ \\
\hline Phallomyces & Batista et al. 1961 & Echinoplaca & Lucking et al. 1997 \\
\hline Psathyromyces & Batista \& Peres 1964 & Tricharia & Lucking ot al. 1997 \\
\hline Pycnociliospora & Batista et al. 1960 & Strigula & $\begin{array}{l}\text { Batista ot al. } 1960 \text {, } \\
\text { Cavalcante ot al. 1972a, } \\
\text { Lucking ot al. } 1997\end{array}$ \\
\hline Pyriomyces & Batista \& Maia 1965a & Byssoloma & $\begin{array}{l}\text { Sérusiaux } 1992, \\
\text { Lucking et al. } 1997\end{array}$ \\
\hline Septoriomyces & Cavalcante et al. $1972 a$ & Phyllobathellum & Locking et al. 1997 \\
\hline Sporocybomyces & Batista \& Maia 1967 & Echinoplace & Looking et al. 1997 \\
\hline Stephosia & Batista \& Maia 1967 & Phyllophiale & $\begin{array}{l}\text { Farkas \& Sipman 1993, } \\
\text { Lücking ot al. } 1997\end{array}$ \\
\hline Tauromyces & Cavalcante et al. 1972a & Gyalectidium & $\begin{array}{l}\text { Sérusiaux } 1992, \\
\text { Lucking ot al. } 1997\end{array}$ \\
\hline
\end{tabular}

Cuadro 2. Géneros anamórficos de líquenes foliícolas descritos por Batista et al. y sus teleomorfos (según Lücking et al. 1997). 
Echinoplaca, Fellhanera, Gyalideopsis, Porina y Tricharia, la diversidad de especies parece ser extremadamente alta (Sérusiaux 1995; Lücking 1997b-c). La mayoría de estas especies aparentemente son raras, así que muchas veces su descubrimiento se basa en colecciones casuales. Otras suelen tener una distribución restringida y requieren inventarios florísticos extensos para su reconocimiento. Un tercer grupo está formado por especies que obviamente tienen su óptimo en ciertas zonas, por ejemplo regiones montañosas. En algunos géneros o familias, como Fellhanera o Trichothelium, olafamilia Gomphillaceae, existe un gran número de nombres viejos, los que hace necesario revisar material tipo antes de describir nuevos taxa (Lücking 1997a-c). Géneros en donde el aumento de especies reconocidas parece ser bajo (por los menos en la región Neotrópica) incluyen Calopadia, Sporopodium, Strigula y Tapellaria. Un caso particular es Gyalectidium, lo cual fue definido en un sentido moderno por Vezda(1979) y Vezda \& Poelt (1987) y por mucho tiempo contenía solamente la especie tipo, $G$. filicinum Müll. Arg. Hoy día, el género se presenta mucho más diverso, particularmente en el Neotrópico (Sérusiaux \& de Sloover 1986; Farkas \& Vezda 1993; Ferraro 1996, y en prep.; Lücking 1997c).

Con respecto a nombres existentes posiblemente sinónimos de otros taxa, son especialmente los géneros y especies publicados por Batista y su grupo que hay que considerar. En 1981, Vobis \& Hawksworth presentaron un resumen de los anamorfos descritos por Batista y sus colaboradores, manifestando que en su opinión la mayoría de estos taxa tiene una base válida. Sin embargo, después varios autores opinaron que por lo menos algunos géneros representan anamorfos de géneros o especies ya conocidos (Sérusiaux 1986, 1992; Farkas \& Sipman 1993), lo cual fue confirmado por estudios recientes del material tipo(Lücking et al. 1997a; vease Cuadro 2). Batista y sus colaboradores mismos eran conscientes de esta situación (Batista \& Maia 1965a: 373): "The proposal of this new genera as real entities is done, until further work disclose the fact that they constitute metagenetic forms of ascolichens." Aún más obvio, Cavalcante et al. (1972a) establecieron los géneros Aulaxina, Lyrommotheca nom. inval. y Strigula como teleomorfos de
Microxyphiomyces, Lyromma y Pycnociliospo$\mathrm{ra}$, respectivamente, así tratando los anamorfos como géneros formales según el ICBN, Art. 59. Esta práctica es común en hongos no lichenizados en donde anamorfos con el estado teleomórfico desconocido son frecuentes y de considerable importancia económica. Sin embargo, Sérusiaux (1992) opina que el Art. 59 no vale para hongos liquenizados y que la práctica de establecer géneros formales en líquenes es incorrecta. En general, la aplicación correcta del articulo en cuestión es poco clara, y el hecho de que es necesario de hacer una decisión biológica si se trata de un hongo liquenizado o no antes de aplicar el reglamento lo hace aún más dificil. En todo caso, no tiene sentido usar géneros formales en líquenes en donde usualmente es posible determinar anamorfos a nivel de género o hasta la especie.

Las especies teleomórficas descritas por Batista y sus colaboradores también fueron poco conocidas hasta la fecha. El género Phragmopelteca (Xavier Filho 1976a) fue puesto en sinonimía con Mazosia (Eriksson 1981), pero Lücking \& Matzer (1996) opinan que más bien podría ser un sinónimo de Porina, lo cual fue confirmado por Lücking et al. (1997a). Además, Batista y sus colaboradores mencionaron varias especies nuevas las cuales nunca fueron validamente publicadas (vease Lücking et al. 1997a). Ejemplos son Arthonia orbicularis Bat. \& J.L. Bezerra nom. inval. (Batista \& Bezerra 1961), Dimerellamacrospora Bat. \& J. L. Bezerra nom. inval. (Bezerra et al. 1970), Opegrapha duckei Bat. nom. inval. (Batista et al. 1961), Porina minuta Bat., J. L. Bezerra \& Cavalc. nom. inval. (Batista et al. 1961), P. oenocarpiBat., Peres \& H. Maia nom. inval. (Batista et al. 1961) o Raciborskiella zollerniae Bat. \& Lima nom. inval. (Batista 1961; Bezerra et al. 1970). También presentaron ampliaciones de distribución considerables para especies como Porina applanata Vain. (Bezerra et al. 1970), P. cerina (Zahlbr.) R. Sant. (Bezerra et al. 1970), P. conica R. Sant. (Batista 1961; Bezerra et al. 1970), P. kamerunensis F. Schill. (Batista et al. 1961; Bezerra et al. 1970; Cavalcante et al. 1972 b) o P. thaxteri R. Sant. (Bezerra et al. 1970). Ninguno de estos reportes pudo ser confirmado(Lücking et al. 1997a). En los ultimos años, muchas especies en géneros los cuales también son frecuentes sobre otros sustratos resultaron pertenecer a géneros diferen- 
tes. El ejemplo más conocido es Bacidia, de la cual muchas especies foliícolas fueron transferidas a otros géneros, como Bacidina (considerado como sinónimo del género Woessia por Sérusiaux 1995), Badimia, Bapalmuia, Barubria, Fellhanera o Loflammia (Vezda 1986, 1990; Sérusiaux 1993). Especialmente en la región Amazónica, pero también en otras áreas, concurren varias especies de "Bacidia" cuya afinidad taxonómica no está bien establecida. Por ejemplo, B. psychotriae (Müll. Arg.) Zahlbr. forma un grupo bien definido junto con Byssoloma wettsteinii (Zahlbr.) Zahlbr. y B. ortizii R. Lücking (Lücking 1995d, 1996b), probablemente relacionado con los géneros Badimia y Fellhanera. Bacidia consimilis (Müll. Arg.) Zahlbr. y B. corallifera R. Lücking, también podrían pertenecer aqui. B. consanguinea (Müll. Arg.) Zahlbr. y B. brasiliensis (Müll. Arg.) Zahlbr. probablemente son relacionadas con Bapalmuia palmularis (Müll. Arg.) Sérus. [sinónimo nuevo: Bacidia rubicunda (Müll. Arg.) Zahlbr., Brasil, Puiggari 1086(G, hólotipo!)]. Stirtonia sprucei R. Sant. y Cryptotheciafarkasiae R. Lücking forman un género propio, ahora llamado Amazonomyces (Lücking et al. 1997a), y Arthothelium cingulatum R. Sant. es relacionado con Eremothecella calamicola Syd. (Ferraro \& Lücking 1997). La especie descrita como Lopadium vulgare Müll. Arg., la cual es muy frecuente en el Neotrópico, demuestra las problemas que a veces existen con la afinidad genérica: fue incluida en Tricharia por Santesson y en Actinoplaca por Vezda \& Poelt, y recientemente transferida al género Gyalideopsis (Lücking 1997c). La familia Gomphillaceae generalmente necesita una revisión a nivel genérico (Lücking 1997c). También son discutidas las relaciones sistemáticas entre los géneros Asterothyrium, Linhartia y Psorotheciopsis dentro de la familia Asterothyriaceae (Henssen \& Lücking, in prep.).

Una posibilidad de estudiar la -taxonomía de especies o dar a conocer conceptos taxonómicos son exsiccatas. Existe una gran cantidad de exsiccatas en donde se distribuyen líquenes foliícolas Neotropicales, por ejemplo Arnold: LICHENES EXSICCATI; Balansa: PLANTES DU PARAGUAY; Cummings: LICHENES BOREALIAMERICANI;Follmann:LICHENESEXSICCATI SELECTIA MUSEOHISTORIAENATURALIS CASSELENSI EDITI; Malme \& Santesson:
LICHENES AUSTROAMERICANI EX HERBARIO REGNELLIANUM; Rehm: ASCOMYCETES; Spruce:LICHENES AMAZONICIET ANDINI LECTI A DOMINI SPRUCE; Vainio:LICHENESBRASILIENSESEXSICCATI; Zahlbruckner et al.: KRYPTOGAMAS EXSICCATASEDITAEA MUSEOPALATINO VINDOBONENSI; Zahlbruckner \& Redinger: LICHENESRARIORIEXSICCATIyespecialmente Kalb: LICHENES NEOTROPICI, Lücking: LICHENES FOLIICOLI EXSICCATI, Sipman: LICHENOTHECA LATINOAMERICANA, Vezda:LICHENESSELECTIEXSICCATIy Vezda: LICHENES RARIORI (vease Lynge 1915; Santesson 1952; Sayre 1969; Farkas \& Sipman 1993;Alava 1993;Lücking 1993a,b, 1995d-g, 1996b).

Florística y biogeografía. El conocimiento florístico varía mucho entre las diferentes países (Fig. 1). Costa Rica es el país mejor investigado hasta la fecha(Kalb \& Vezda 1988a,b, 1990; Lücking 1992a, 1995a, 1996d, 1997a-c; Lücking \& Matzer 1996). Paises con un conocimiento más o menos bueno incluyen Guatemala (Barillas \& Lücking 1992), Cuba(Vezda 1984), Colombia (Nowak \& Winkler 1970, 1975; Sipman 1990a), las Guayanas (Aptroot \& Sipman 1993; Sipman 1996), Brasil (Santesson 1952; Kalb \& Vezda 1988a, b, 1990), Uruguay (Osorio 1970-1992) y Argentina (Ferraro 19821996; Sérusiaux \& de Sloover 1986; Ferraro \& Vezda 1989). Por otro lado, comparado con su diversidad teorética en líquenes foliícolas, países como Mexico, Honduras, la mayoría de las Antillas, Ecuador, Venezuela o las Guayanas se presentan relativamente mal investigados. Aún peor es la situación en Nicaragua, Panamá, Perú, Bolivia y Chile, donde se conoce practicamente nada. Justamente Panamá tiene una gran tradición en estudios florísticos de plantas superiores y, junto con Guatemala y Costa Rica, es el país mejor recolectado en América Central (Prance 1977). En otros países, como Nicaragua, Perú y Bolivia, la situación en plantas superiores es comparable con la de los líquenes foliícolas y criptógamas en general.

Correspondientemente, existen muy pocos trabajos biogeográficos. Algunos se limitan a mencionar la distribución de especies (Kalb \& Vezda 1988a, 1990), mientras otros investigaron patrones de distribución y afinidades biogeográficas 


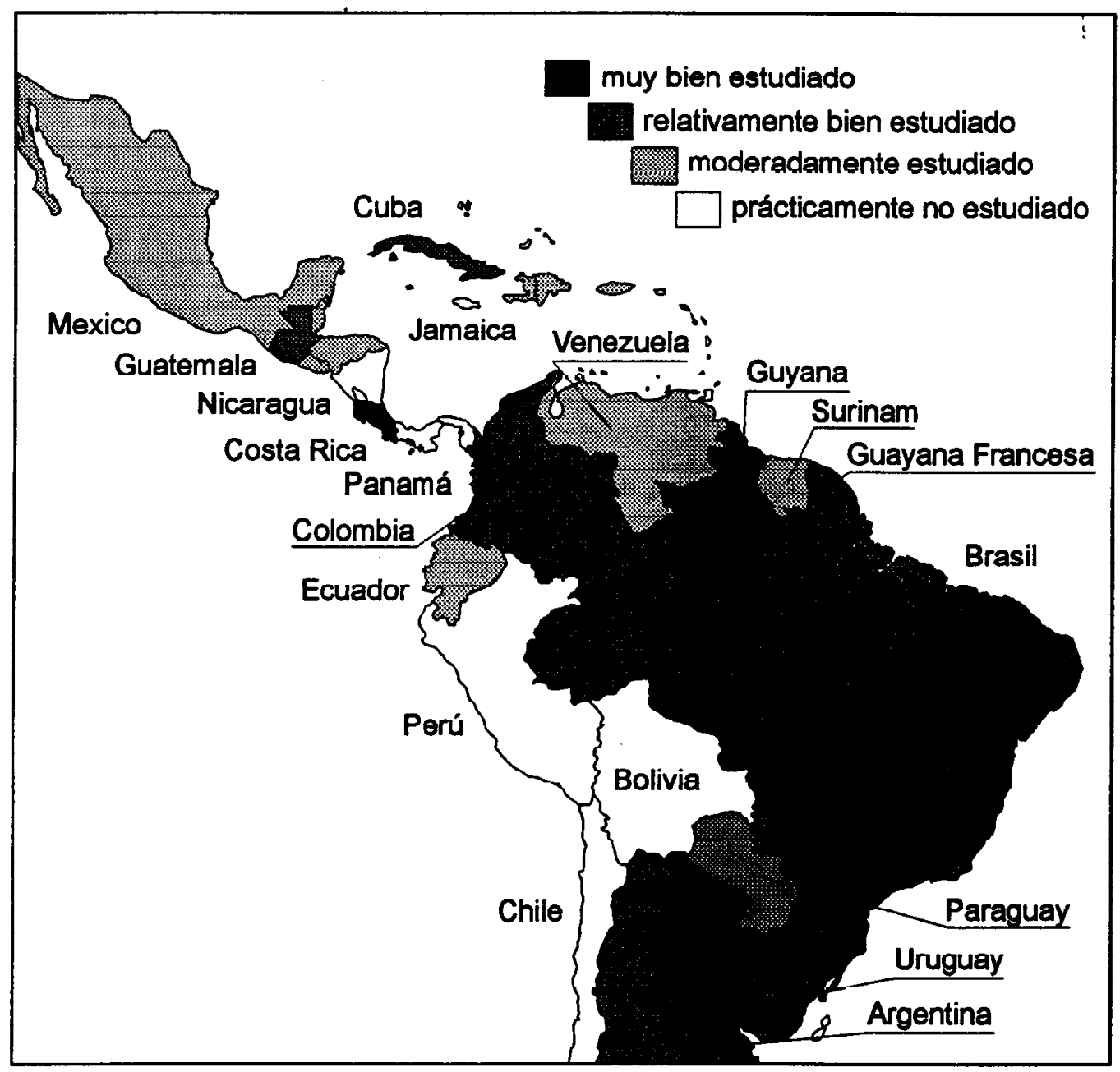

Fig. 1. Estado actual de investigaciones sobre líquenes foliícolas en los países Neotropicales (basado en el número de especies reportadas y su relación con la diversidad estimada).

(Lücking 1990; Lücking \& Lücking 1995; Becker \& Lücking 1995; Lücking etal. 1997b). Generalmente, las ideas sobre la distribución de líquenes foliícolas son muy poco claras, una situación semejante a la de otras criptógamas (Grolle 1968). Conclusiones comolas de Schell \& Winkler(1980), que comparan patrones de distribución de líquenes foliícolas con las de bromelias en una área limitada, son por lo tanto inseguro y deben ser consideradas con mucho cuidado.
Ecología. Mientras que los primeros estudios ecológicos fueron realizados en el Paleotrópico, estudios modernos se hicieron en su mayoría en el Neotrópico, también aplicando por primera vez métodos cuantitativos, como el análisis de asociaciones. Temas ecológicos particularmente investigados en el Neotrópico incluyen las preferencias de habitat y altura (Nowak \& Winkler 1970; Sérusiaux \& de Sloover 1986; Lücking 1992b-c, 
1995c, 1996c), las preferencias de microhabitat y del forófito (Barillas et al. 1993; Coley et al. 1993; Lücking 1994b) y la estratificación vertical desde el sotobosque al dosél (Montfoort \& Ek 1990; Lücking 1995b; Sipman 1996), tanto como la biodiversidad y sus mecanismos de mantenimiento (Lücking 1995c). También fueron realizadas investigaciones sobre la successión (Schell \& Winkler 1980; Hartmann 1993), aunque menos detalladas como las de Conran \& Rogers (1983) Rogers \& Barnes (1986) y Rogers (1989) en Australia.

Según estos estudios, los líquenes foliícolas tienen su mayor diversidad en los bosques lluviosos de bajura. El número de especies es menor en bosques montañosos y en vegetación perturbada y mucho menor en bosques semideciduos o áreas secas (Sérusiaux \& de Sloover 1986; Lücking 1992b-c, 1995c). Hasta 200 especies o más pueden ocurrir en una localidad particular, lo que significa una concentración enorme de la diversidad (Lücking 1994b, 1995c). Dentro de una localidad, la distribución de especies depende del microclima, con tres asociaciones principales en el sotobosque, en claros y en el dosél. La influencia del forófito parece ser menos pronunciada, pero los resultados son controvertidos (Barillas et al. 1993; Coley et al. 1993; Lücking 1994). El desarollo de la liquenoflora foliícola en una hoja es rapido en los bosques lluviosos y puede alcanzar su estado de diversidad máxima después de 2-3 años (Hartmann 1993; Lücking 1995c).

Aplicaciones. El uso de líquenes como bioindicadores ambientales tiene una gran tradición en los países templados, pero también existen intentos de establecer aplicaciones en los trópicos (Vareschi 1953; Sipman \& Harris 1989; Wolseley 1991; Wolseley \& Aguirre-Hudson 1991; Galloway 1992; Wolseley et al. 1994). Recientemente se ha demostrado el potential de líquenes foliícolas como indicadores de biodiversidad, de la dinámica de la vegetación, y de factores ambientales como la altura, el grado de perturbación y el microclima (Lücking 1995c, 1996c). Comparados con otros organismos, los líquenes foliícolas tienen varias ventajas que incluyen la alta susceptibilidad ante factores microclimáticos, el crecimiento bidimensional, el buen conocimiento taxonómico, la facil recolecta y documentación y el desarollo rapido de las comunidades y su gran diversidad, particularmente en bosques de bajura donde otras epífitas generalmente son escasas.

Elementos característicos de la región Neotrópica y sus subregiones, con un análisis biogeográfico preliminar

En esta sección se quiere dar en primer lugar una comparación entre las tres mayores regiones tropicales, evaluando asíla importancia de la región Neotrópica y tratando un análisis biogeográfico preliminar. Luego se presentan elementos característicos Neotropicales entre los líquenes foliícolas y se comparan las diferentes subregiones biogeográficas dentro de la región Neotrópica. Las figuras presentadas se basan principalmente en la monografía de Santesson (1952), en la lista presentada por Farkas \& Sipman (1993), en publicaciones recientes y en observaciones del autor.

Un análisis biogeográfico es algo difícil porque el desarrollo del conocimiento taxonómico y florístico de líquenes foliícolas es rapido, y patrones de distribución son mucho menos pronunciados que en plantas superiores (Prance 1977). Mientras que Santesson (1952) acepta 236 taxa de líquenes foliícolas, cuarenta años después Farkas \& Sipman (1993) incluyen más del doble en su lista. Desde entonces fueron descritas unas 100(!) especies más, y también cambió el conocimiento de la distribución de varios taxa (Aptroot \& Sipman 1993a, b; Sipman 1993a, b; Farkas \& Vezda 1993; Sérusiaux 1993, 1995; Vezda 1994; Kalb \& Vezda 1994; Malcolm \& Vezda 1994, 1995; Lücking 1994, 1995a-b, 1997a-c; Becker \& Lücking 1995; Lücking \& Matzer 1996; Aptroot et al. 1997). Sin embargo, los porcentajes de elementos biogeográficos quedaron relativamente constantes. Por ejemplo, especies exclusivamente Neotropicales hacen un 20-25\% en la monografía de Santesson (1952) tanto como en la lista de Farkas \& Sipman (1993) y actualmente.

Diversidad de especies. El número de especies varía en las tres regiones tropicales. La diversidad más alta se encuentra en la región Neotrópica, con alrededor de 350 especies. En segundo lugar está Australasia tropical con unas 290 especies, seguido por África tropical que tiene alrededor de 


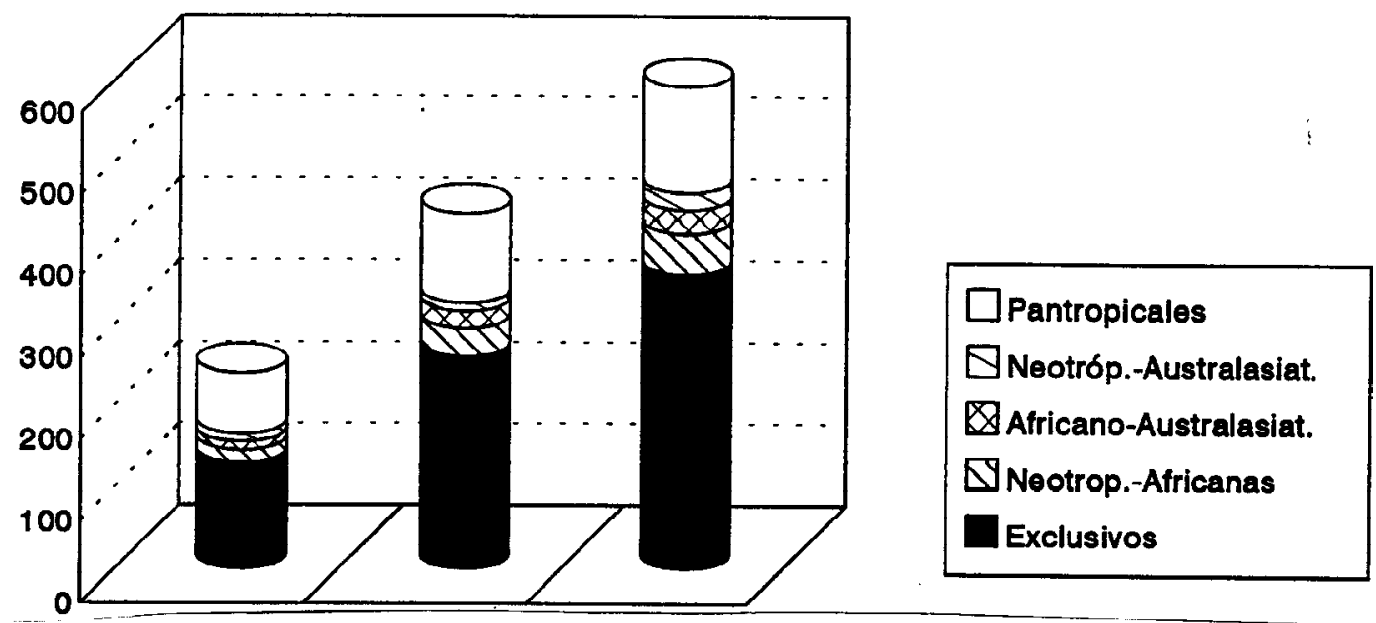

Santesson 1952 Farkas \& Sipman 1993 Estado actual 1997.

Fig. 2. Número aproximado de especies de líquenes foliícolas y tipos de distribución, según Santesson (1952), Farkas \& Sipman (1993) y el estado actual 1997. El mayor porcentaje es contribuido por especies siendo exclusivas para el Neotrópico, África o Australasia.

260 especies. En esto hay que considerar que África tropical parece ser mejor investigada que Australasia y la región Neotrópica, especialmente porlos trabajos de Vezda (1973, 1974, 1975a, 1975b, 1980,1982, 1987)y Sérusiaux (1977, 1978, 1979a-b, 1983), así que las diferencias entre las tres regiones podrían presentarse aún más pronunciadas en el futuro. Por ejemplo, colecciones recientes de Papua Nueva Guinea demuestran una diversidad de líquenes foliícolas mucho más alta que la considerada antes (Aptroot et al. 1997).

Esta situación es comparable con la de plantas superiores en donde se estima que el Neotrópico tiene más que el doble de especies que el Paleotrópico, aunque es la región menor conocida (Prance 1977; Gentry 1982a, 1988). La pobreza de especies, especialmente epífitas, de África tropical se explica principalmente por el empeoramiento climático extendido durante el Pleistoceno y el Terciario superior (Raven \& Axelrod 1974; Burger 1980; Gentry 1988). Esto parece ser valido también para los líquenes foliícolas, que tienen su mayor diversidad en África tropical en los bosques montañosos (500-2000 m) o en bosques de bajura que posiblemente representan refugios para el bosque lluvioso durante períodos secos (Lücking et al. 1997b). Por lo tanto, la riqueza de especies en la región Neotrópica es probablemente debida a un clima más constante, con perturbaciones menos pronunciadas, que más bien provocaron la evolución de nuevas especies, como está formulado en la teoría de los refugios (Prance 1982).

Tipos de distribución y afinidades biogeográficas entre las regiones tropicales mayores. Se pueden distinguir siete tipos principales de distribución de especies entre los líquenes foliícolas: (1) Pantropical; (2) Neotropical-Africano; (3) Neotropical-Australasiático; (4) Africano-Australasiático; (5) Neotropical; (6) Africano; (7) Australasiático. Alrededor de 130 especies tienen una distribución pantropical, que es un $23 \%$ de todas las especies analisadas (Fig. 2).

Actualmente se conocen unas 40 especies con distribución Neotropical-Africana, mientras 20 especies son reportadas de la región Neotrópica y Australasia tropical, y alrededor de 25 taxa de África y Australasia tropical. El gran número de especies con una distribución amplia (conocidas al menos de dos regiones principales) es un fenómeno particular de plantas inferiores. Grolle (1968:562), por ejemplo, observa una"...Zunahme der Arealweite der Arten und Gattungen zu den Pflanzenstämmen niedrigen Organisationsgrades hin." En contrario, en plantas superiores existen pocas especies que tienen una distribución tan amplia que no sea causada por la influencia del hombre, y todavía a nivel de géneros el porcentaje es menor que el de especies en líquenes foliícolas 


\begin{tabular}{|c|c|c|c|}
\hline 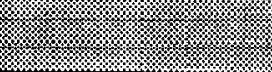 & 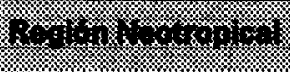 & (2), & 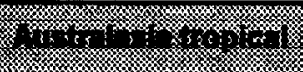 \\
\hline Región Neotropical & $\longrightarrow$ & $\begin{array}{l}0.58 \\
0.59 \\
0.68\end{array}$ & $\begin{array}{l}0.50 \\
0.49 \\
0.53\end{array}$ \\
\hline Africa tropical & $\begin{array}{l}0.58 \\
0.59 \\
0.68\end{array}$ & $\longrightarrow$ & $\begin{array}{l}0.56 \\
0.57 \\
0.64\end{array}$ \\
\hline Australasia tropical & $\begin{array}{l}0.50 \\
0.49 \\
0.53\end{array}$ & $\begin{array}{l}0.56 \\
0.57 \\
0.64\end{array}$ & 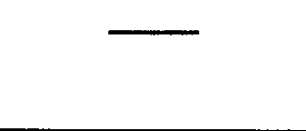 \\
\hline
\end{tabular}

Cuadro 3. Afinidades biogeográficas entre las tres regiones tropicales, según el índice de afinidad de Sørensen (1948). Primera fila (en negrita): Estado actual 1997; segunda fila: según Farkas \& Sipman 1993; tercera fila: según Santesson 1952.

(Gentry 1988). Esto significa un problema para un análisis biogeográfico en líquenes foliícolas y criptógamas en general, porque usualmente las regiones biogeográficas mundiales se basan en la distribución de plantas superiores, y es difícil adaptar la situación de plantas inferiores a este sistema (Grolle 1968).

Tanto el Neotrópico como Australasia tropical tienen un alto número de especies exclusivas, con alrededor de $40 \%$ de su número total respectivo, mientras que en África tropical, este porcentaje es mucho menor(Fig. 3), explicando partialmente el número menor de especies encontradas en esta región. Se observa que el porcentaje de especies exclusivas para una región ha aumentado levamente desde la monografía de Santesson (1952), mientras que el porcentaje de especies pantropicales bajó (Fig. 3). Esto se debe partialmente al aumento del conocimiento de regiones particulares durante los últimos años, pero también al hecho de que muchas especies han sido descritas muy recientemente (durante los últimos cinco años), y falta verificar si realmente son exclusivas para una región particular, o sea macroendémicas, o más bien tienen una distribución más amplia pero no fueron reconocidas en otras regiones. Si se compara las tres regiones tropicales mayores con el indice de similaridad de Sørensen (1948), la afinidad biogeográfica entre la región Neotrópica y África tropical es mayor que entre África tropical y Australasia tropical(Cuadro
3). Tambíen el índice de similaridad entre la región Neotrópica y Australasia tropical es alto. Un patrón similar, si se hace un análisis a nivel de géneros, se encuentra en plantas superiores (Delevoryas 1973; Gentry 1988).

Se supone que la estrecha afinidad biogeográfica entre la región Neotrópica y África tropical se basa en una conexión directa entre los dos continentes antes de 90-100 millones de años (Dietz \& Holden 1970; Baker 1973), pero esta hipótesis es discutida (Martin 1968). Algunos autores piensan que patrones de distribución disyuncta o amplia se deben más bien a la dispersión a larga distancia (Thorne 1973; Smith 1973; Tibell 1994). En todo caso, la relación biogeográfica entre la región Neotrópica y África tropical es obvia en los líquenes foliícolas y quedó demostrada por varios resultados (Becker \& Lücking 1995; Lücking et al. 1997b). Ejemplos de especies con este patron incluyen Arthonia accolens Stirt., Asterothyrium argenteum Müll. Arg., A. monosporum Müll. Arg., Aulaxinaminuta R. Sant., A. quadrangula(Stirt.)R. Sant.,Badimiadimidiata(Babingt.exLeight.) Vezda (Fig. 4A), Fellhanera lisowskii (Vezda) Vezda, Mazosia praemorsa (Stirt.) R. Sant. (Fig. 5C), Psorotheciopsis albomaculans (Rehm) R. Sant., P. premneella (Müll. Arg.) R. Sant., Strigula schizospora R. Sant. o Tricharia urceolata (Müll. Arg.) R. Sant.

El gran número de especies con distribución Neotropical-Australasiática no parece explicable 
Neotrópico

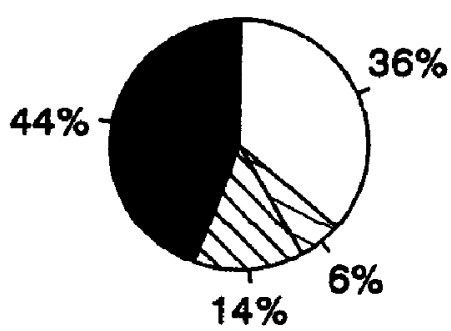

Africa tropical Australasia tropical

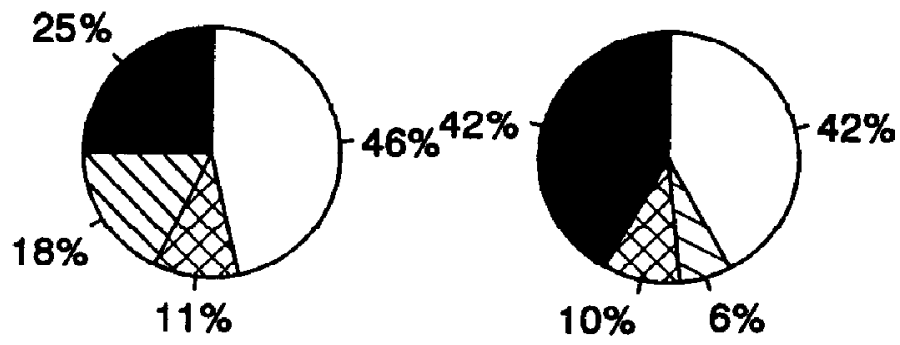

Estado actual 1997

Neotrópico

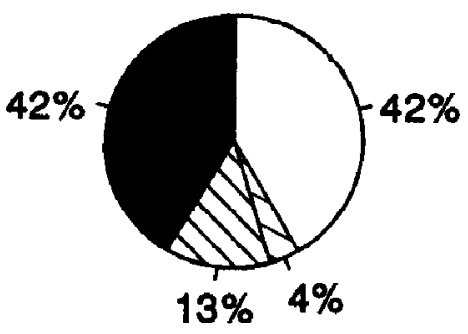

Africa tropical Australasia tropical

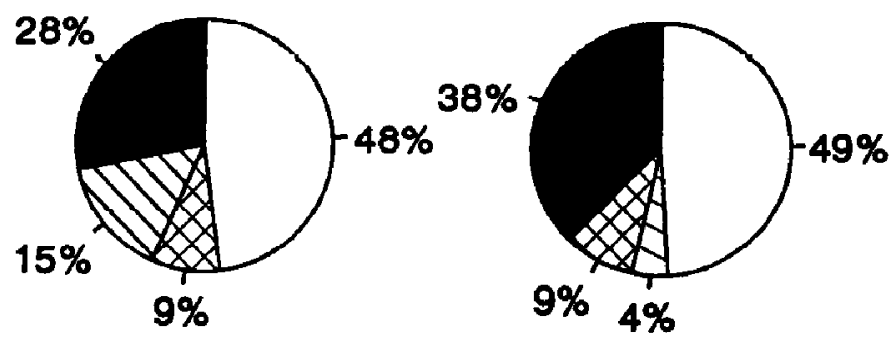

Farkas \& Sipman 1993

Neotrópico

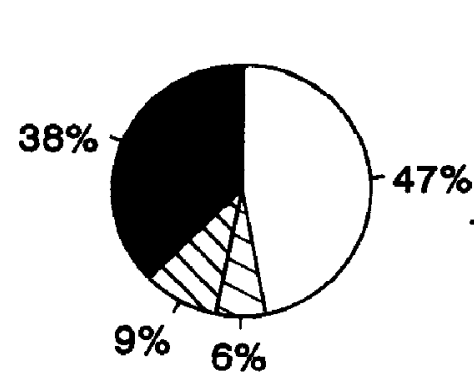

Africa tropical

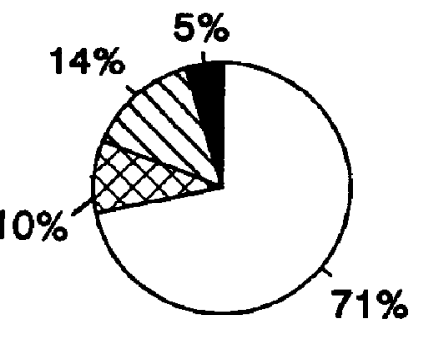

Santesson 1952

Australasia tropical

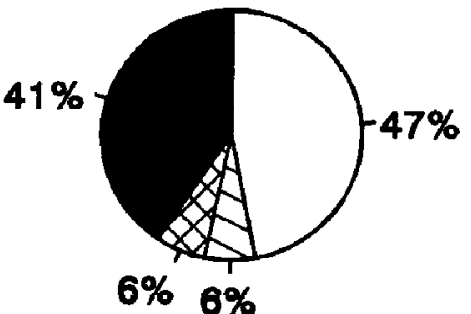

$6 \% 6 \%$

Fig. 3. Porcentaje aproximada de tipos de distribución en las tres regiones tropicales, según Santesson (1952), Farkas \& Sipman (1993) y el estado actual 1997. Por la signatura vease Fig. 2. 
a primera vista. Algunos autores piensan que este tipo de distribución, que también ocurre en algunos géneros de líquenes no foliícolas, es un artefacto, y que las especies no han sido encontradas en África por la falta de recolecciones, o están extinctas. Esto parece plausible ya que la gran mayoría de líquenes foliícolas con esta distribución es típica para los bosques tropicales lluviosos de bajura, que han sido ampliamente reducidos en África durante el Pleistoceno. Ejemplos incluyen Badimia galbinea (Kremp.) Vezda, B. pallidula (Kremp.) Vezda, Caleniaphyllogena(Müll. Arg.) R. Sant. (Fig. 4B), o Tricharia santessonii D. L. Hawksw. Por otro lado, no se puede excluir la posibilidad de que especies migraron directamente entre el Neotrópico y Australasia, por las islas Pacificas. La afinidad entre África y Australasia tropical se debe principalmente a las rutas de dispersión recientes (Gentry \& Dodson 1987), en África resultando en la mezcla actual de especies con distribución Neotropical-Africana y AfricanaAustralasiática (Lücking et al. 1997b).

Elementos característicos de la región Neotrópica y sus subregiones. La región Neotrópica tiene el mayor número de especies exclusivas. Entre ellas, se encuentran taxa como Actinoplaca strigulacea Müll. Arg., Arthonia mira R. Sant., Bacidia brasiliensis (Müll. Arg.) Zahlbr. (Fig. 5A), B. psychotriae (Müll. Arg.) Zahlbr., Calenia triseptataZahlbr., Chroodiscus neotropicus Kalb \& Vezda,Echinoplaca atrofusca R. Sant.(Fig.5B), Eremothecella cingulata (R. Sant.) L.I. Ferraro \& $R$. Lücking, Gyalideopsis vulgaris (Müll. Arg.) Vezda \& Poelt, Mazosia tumidula (Stirt.) Müll. Arg., Phyllobathelium epiphyllum (Müll. Arg.) Müll.Arg. (Fig. 5D), Tricharia farinosa R. Sant.o Trichothelium argenteum R. Lücking \& L. I. Ferraro. Algunas especies están estrechamente relacionadas con otras de África tropical, como Fellhanera stanhopeae (Müll. Arg.) R. Lücking, Lumbsch \& Elix vs. F. lambinonii (Sérus.) R. Lücking \& Sérus. o Phylloblastia amazonia Kalb \& Vezda vs. P. dolichospora Vain. (Fig. 5C-D). Otras especies son muy comunes en la región Neotrópica pero también ocurren en otras regiones, como Arthonia leptosperma (Müll. Arg.) R. Sant., Chroodiscus coccineus (Leight.) Müll. Arg., Coccocarpia domingensis Vain., Mazosia rotula (Mont.) A. Massal., Porina rugosa Kalb \&
Vezda o Strigula schizospora R. Sant.

A nivel de géneros, la situación es más complicada, por los continuos cambios sistemáticos. En la lista de Farkas \& Sipman (1993), alrededor de 50 géneros aparentemente son exclusivos para el Neotrópico o para el Paleotrópico. La mayoría de estos se refiere a Batista y sus colaboradores, de los cuales solo quedan Amazonomyces y Lyromma (Lücking et al. 1997). De los otros géneros, Clathroporina es probablemente un sinónimo de Porina. El género Pseudogyalecta fue puesto en sinonimía con Badimia (Lücking \& Vezda 1995; Vezda \& Lücking 1995), y el género Semigyalecta aparentemente es un hongo no liquenizado (Kalb \& Vezda 1994; Thor et al. 1997). La única especie foliícola del género Pleurotrema actualmente pertenece a Anisomeridium (Harris 1995; Thoret al. 1997), y los mismo podría ocurrir con el género Porinula (Harris 1995). La separación de los géneros Actinoplaca y Echinoplaca no está bien establecida. Algunos géneros, como Megalospora, Myriotrema, o Phaeographis, contienen especies (facultativamente) foliícolas en el Paleotrópico pero también ocurren en el Neotrópico con especies no folíícolas. Con esto, solo quedan pocos géneros con especies principalmente foliícolas que podrían ser exclusivos para el Neotrópico o para otras regiones tropicales, e.g. Actinoplaca y Amazonomyces para el Neotrópico, y Badimiella y Podotara para Australasia.

La región Neotrópica puede ser dividida formalmente en seis subregiones mayores (Sick 1968; Gentry 1982; Gradstein 1987; A. Lücking 1995; vease Fig. 6): (1) Norteamérica (incluyendoMexico $y$ partes tropicales y subtropicales de Estados Unidos), (2) América Central (desde Guatemala al norte de Panamá), (3) las islas Pacíficas (principalmente Isla del Coco, Islas Galápagos), (4) las islas Caribeñas o las Antillas (desde Cuba a Puerto Rico, y desde Guadalupe a Trinidad), (5) Suramérica Oeste (principalmente los Andes de Colombia, Venezuela, Ecuador, Peru, Bolivia y Chile),y (6) SuraméricaEste (principalmente Brazil y sus alrededores: Venezuela, las Guayanas, Paraguay, Argentina y Uruguay). Aunque en líquenes folíícolas, los patrones de distribución son mucho menos claros que en plantas superiores o briófitos (Gradstein 1987,1994), especialmente a escala menor(Grolle 1968), es posible correlacionar 

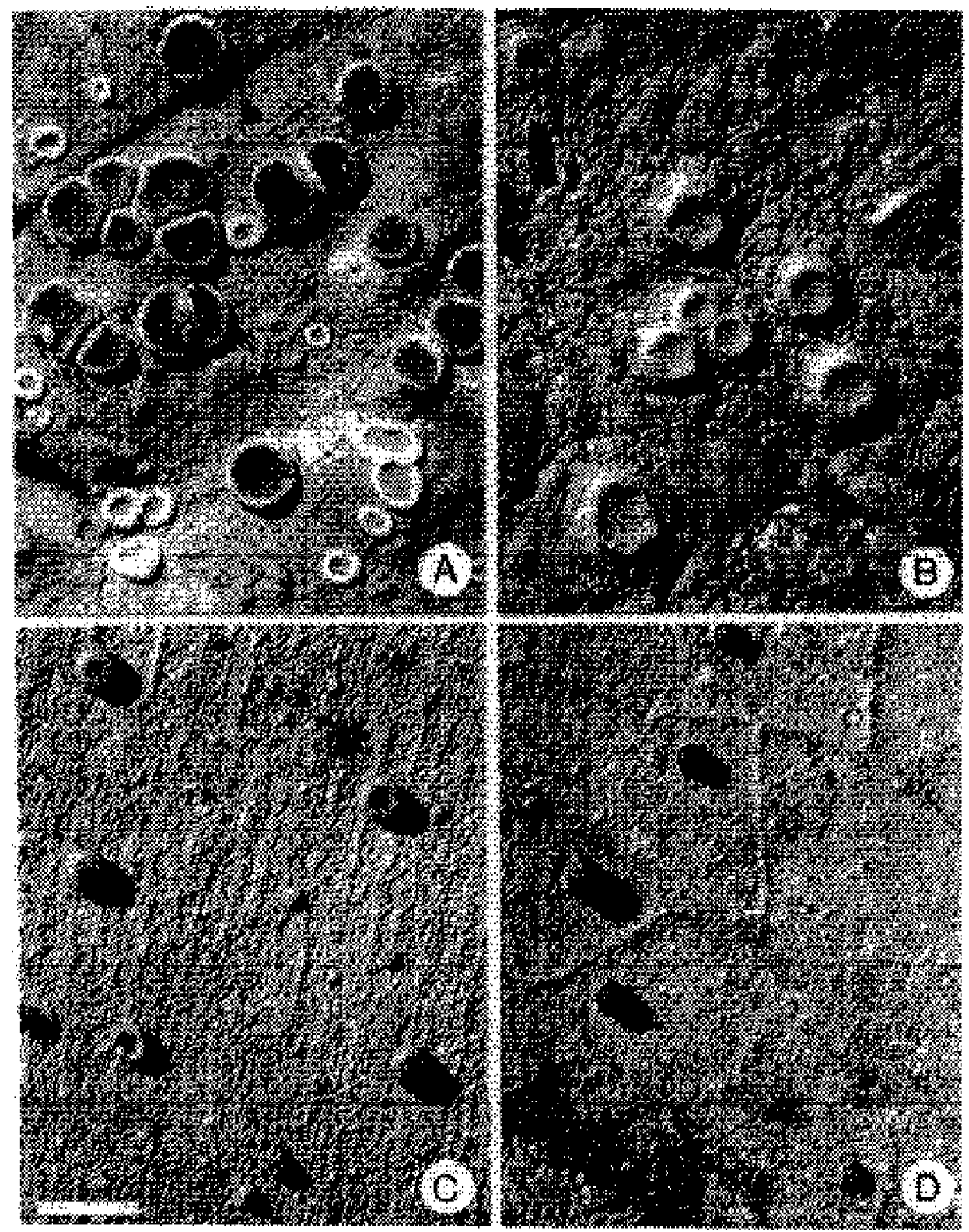

Fig. 4. (A) Badimia dimidiata (Neotrópico y África). (B) Calenia phyllogena (Neotrópico y Australasia). (C) Phylloblastia amazonica (Neotrópico). (D) P. dolichospora (Paleotrópico). 

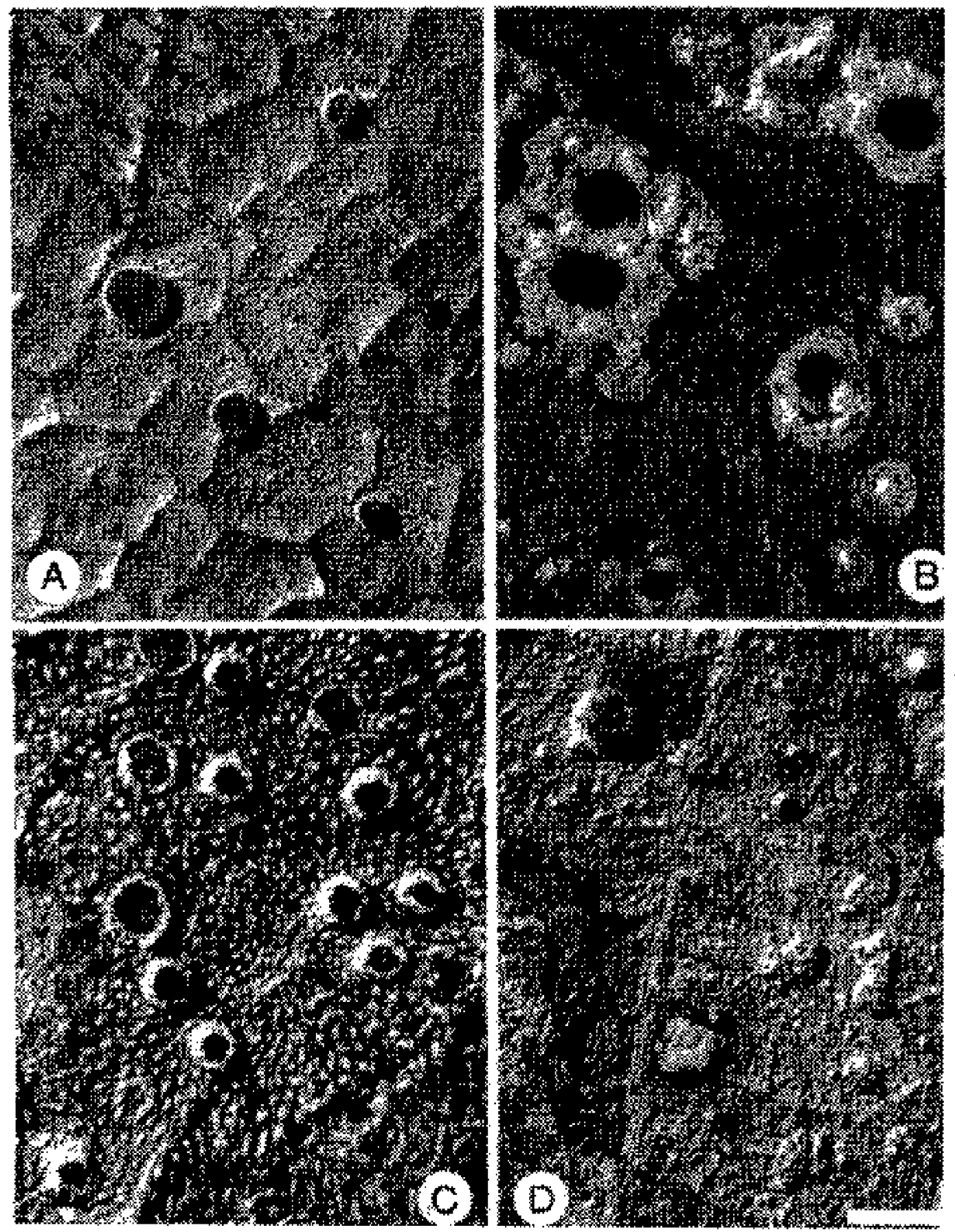

Fig. 5. (A) Bacidia brasiliensis (Neotrópico). (B) Echinoplaca atrofusca (Neotrópico). (C) Mazosia praemorsa (Neotrópico y África). (D) Phyllobathelium epiphyllum (Neotrópico). 


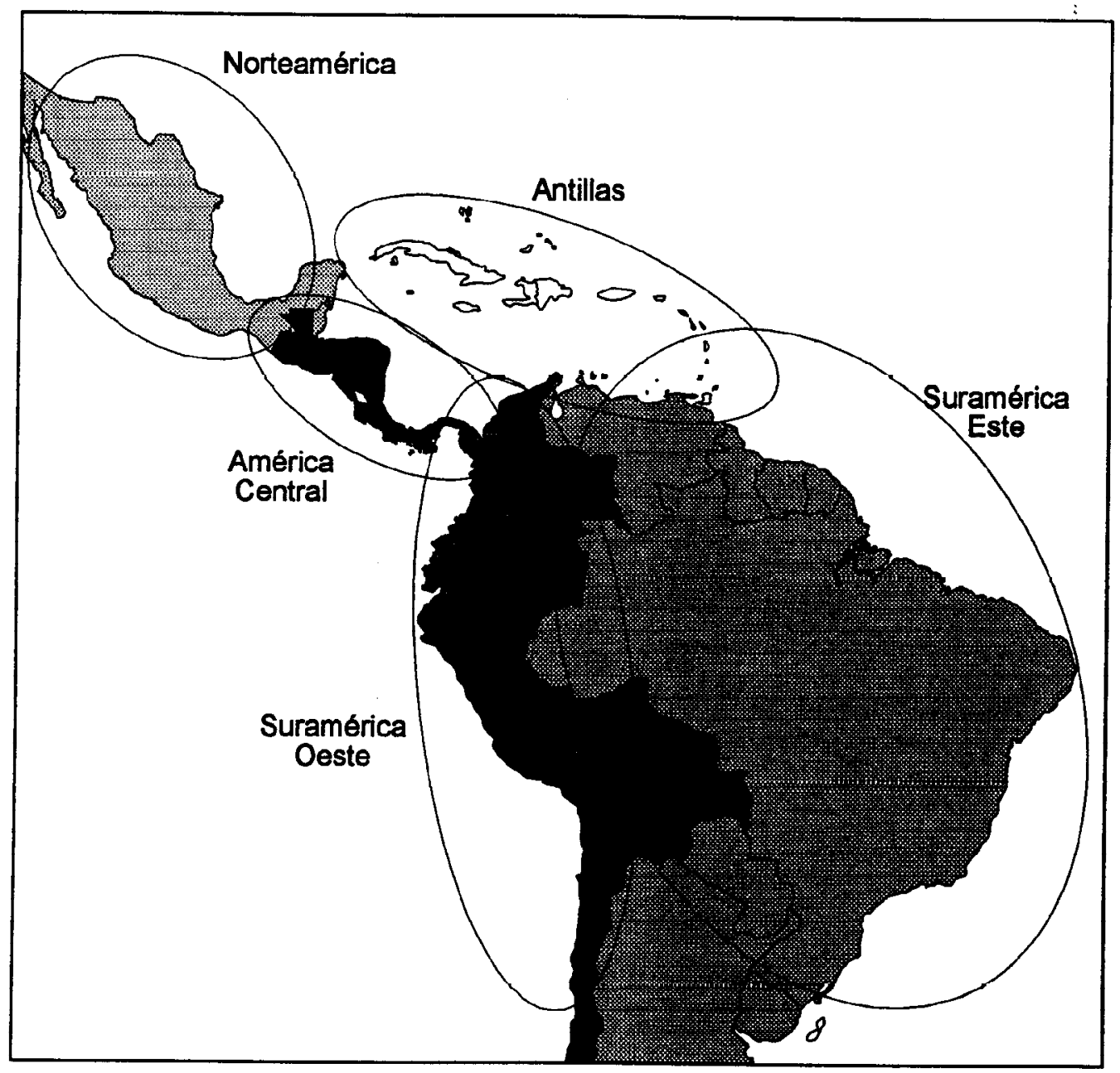

Fig. 6. Las subregiones geográficas mayores en los Neotrópicos y sus correlaciones con límites políticos. Para los nombres de los paises, vease Fig. 1.

por lo menos la distribución de algunas especies bien conocidas con estas subregiones.

El elemento más importante parece ser el de Suramérica Este, lo cual se refiere principalmente a la región Amazónica, desde el Nordeste de Brasil al Este de Colombia, Ecuador, y Perú. Varias especies solo se conoce de esta región, pero pueden serfrecuentes en sitios apropriados: Bacidia consanguinea (Müll. Arg.) Zahlbr., Bacidia psychotriae (Müll. Arg.) Zahlbr., Byssoloma amazonicum Kalb \& Vezda,B.polychromum(Müll. Arg.) Zahlbr., Stirtonia sprucei R. Sant. (Fig. 7A) o Lecidea trailiana Müll. Arg. Muchas de estas especies se encuentran hasta el Oriente de Colombia, Ecuador y Perú pero no ocurren en el vertiente Pacífico de estos países, indicando que los Andes forman un límite natural. Especies que no son restringidas a la región Amazónica pero muestran una frecuencia particularmente alta en esta área son por ejemplo Mazosia pilosa Kalb \& 

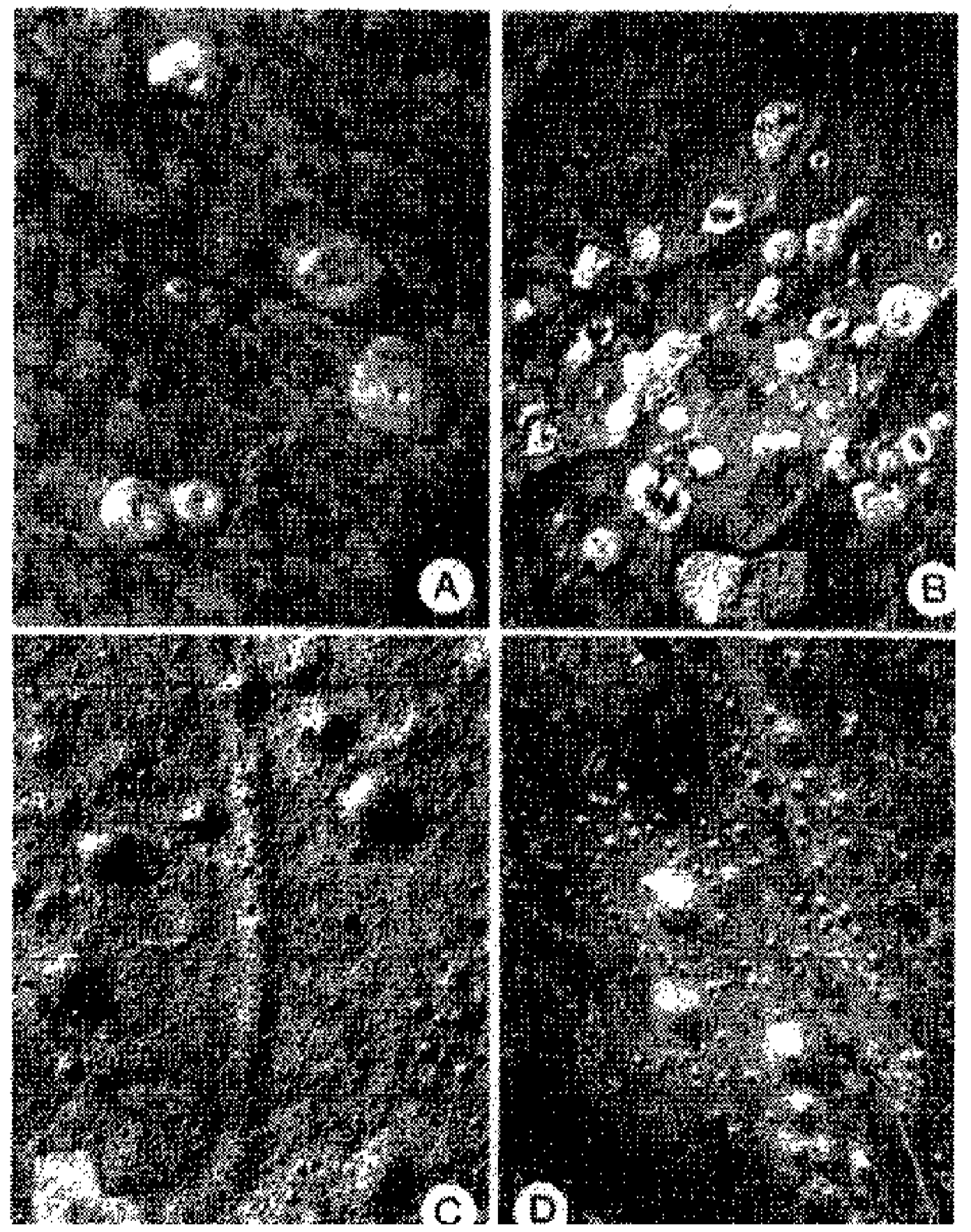

Fig. 7. (A) Stirtonia sprucei (Suramérica Este).(B) Cryptotheciafarkasiae (Suramérica Oeste y América Central). (C) Strigula multipunctata (Suramérica y Antillas). S. viridis (América Central). 
Vezda, M. praemorsa (Stirt.) Müll. Arg., M. rubropunctata R. Sant. y M. tumidula (Stirt.) R. Sant.; aparentemente, Amazonia es una de las regiones de evolución principal de este último género (Lücking \& Matzer 1996).

Varias especies suelen estar restringidas a Suramérica Oeste y América Central y supuestamente representan elementos del Chocó (Gentry 1982; Gradstein 1987) que probablemente es la región más rica en especies de todo el Neotrópico (Prance 1977; Gentry 1986). Cryptothecia farkasiae R. Lücking (Fig.7B), Porinaumbilicata(Müll. Arg.)F. Schill., P. barvica R. Lücking y Trichothelium asplundii R. Sant. son representantes de este grupo. Tres de estas son especies principalmente montañosas, mientras que C. farkasiae es una especie de bajuras. Es interesante que esta especie tiene una pareja estrechamente relacionada en Stirtonia sprucei que solo ocurre en la región Amazónica(Sipman, com. pers. 1995).

Pocas especies son reportadas exclusivamente de América Central o Norteamérica, por ejemplo Calenia aggregata R. Sant. y Strigula viridis (R. Lücking) Harris (Fig. 7D). S. viridis es relacionada con S. multipunctata (G. Merrill ex R. Sant.) Harris (Fig. 7C), que ocurre en Suramérica Este y de las Antillas. Especies que se conocen de América Central y de las Antillas incluyen Calopadia perpallida (Nyl.) Vezda, Fellhanera santessonii Barillas \& R. Lücking, Porina cubana Vezda o Badimiatuckermanii(R.Sant.)R.Lücking,Lumbsch \& Elix. Laúltima no fue encontrado recientemente en el Neotrópico peroes común en Nueva Caledonia (Lücking 1995c, g) y también fue encontrada en Papua Nueva Guinea (Sérusiaux, com. pers. 1996). Especies aparentemente endémicas son también raras. Casos que tal vez podrían ser confirmados son por ejemplo Badimia montoyana R. Lücking, Dimerellaminima(Müll. Arg.) R. Sant. oTricharia paradoxa R. Lücking, reportadas de Costa Rica y de la Isla del Coco, respectivamente.

Aunque los patrones de distribución de muchas especies están poco claros, parece que la región Amazónica es uno de los centros de evolución de líquenes foliícolas. Aunque el número de especies microendémicas es actualmente mayor en algunas otras regiones, el número de especies macroendémicas confirmadas en la región Amazónica es alta. Esta hipótesis es apoyado por el hecho de que la mayoría de las especies tiene su óptimo ecológico en los bosques lluviosos de bajura y por el alto número de especies que principalmente ocurren en esta subregión geográfica (Lücking 1995c, 1996b; Lücking \& Matzer 1996). Es interesante que aparentemente no hay indicaciones para un efecto pronunciado de especiación en las Antillas, comparable con plantas superiores, aunque esta región todavía es poco conocida.

\section{Conclusiones y perspectivas}

Considerando el estado actual de investigaciones sobre líquenes foliícolas, los cuales sin duda son el grupo mejor conocido entre todas las criptógamas no vasculares tropicales, se puede constatar que todavía falta mucho para obtener un inventario completo en el Neotrópico. Actualmente, se puede observar un aumento en las actividades científicas que, a pesar de todo, representan solamente pequeñas contribuciones en el camino a esta meta. Trabajos en progreso incluyen estudios florísticos de varios regiones, como Guatemala [R. Barillas, R. Lücking], Costa Rica [R. Lücking], Colombia [H. Sipman], Ecuador [L. Arvidsson, E. Sérusiaux, R. Lücking], las Guayanas [A. Aptroot, H. Sipman, R. Lücking], Brasil [K. Kalb, R. Lücking], o Argentina [L. Ferraro]. En el marco de las series llamadas Flora Neotropica y Flora of the Guianas, diversas monografías están previstas a salir en los proximos años, comprendieno las familias Asterothyriaceae, Gomphillaceae, Pilocarpaceae y Ectolechiaceae, tanto como los géneros Mazosia y Trichothelium [E. Farkas, K. Kalb, R. Lücking, M. Matzer, G. Thor, A. Vezda].

Es tambíen necesario crear una base más precisa para estudios biogeográficos. Un posible proyecto que fue discutido durante el IAB \& IAL Symposium on Foliicolous Cryptogams en Eger es el inventario detallado y la comparación de tres bosques tropicales situados en el Neotrópico, en África tropical y en Australasia tropical cada uno. Estudios ecológicos están siendo planeados especialmente en Costa Rica y Guayana France$s a$, incluyendo un proyecto de exploración del dosél de los bosques con un nuevo sistema llamado COPAS (Gottsberger \& Döring 1995; Gottsberger et al. 1995; Lücking 1995e). Tambíen hay que 
considerar que todavía faltan muchos inventarios y verificaciones de datos ecológicos, por ejemplo la zonación altitudinal, la correlación con tipos de vegetación y la preferencia de microhabitats en diferentes regiones.

Precisamente estas últimas áreas son una buena posibilidad para contribuciones de liquenólogos locales. Sin embargo, existen varios problemas relacionadas con esto. Es obvio que la gran mayoría de las colecciones de líquenes foliícolas están depositadas en herbarios Europeos, especialmente en Genf [G], Berlin [B] y München [M], una situación preocupante que es semejante, aunque menos pronunciada, en plantas superiores (Prance 1977). Entre los herbarios de la cuales fue estudiado material por Santesson (1952), solo tres están situados en el Neotrópico: en Mexico [LIL], Brasil [RB] y Argentina [LPS]. Actualmente, hay pocos herbarios Neotropicales en donde las colecciones de líquenes foliícolas reflejan la diversidad actual de la región y el grado de colección, por ejemplo San José [CR] en Costa Rica, Recife [URM] en Brasil, y Corrientes [CTES] en Argentina. Esta situación tiene razones historicas, pero también se debe a la opinión de muchos recolectores y científicos extranjeros de que los herbarios tropicales noestán bien organizados, especialmente con respecto al mantenemiento de especímenes, y por eso raramente depositan sus recolecciones aya. Además, la mayoría de los taxónomos están situados afuera del Neotrópico, lo mismo como sucede en las plantas superiores (Gentry 1977). Según Feuerer (1995), existen menos de 20 liquenólogos con enfasís en la taxonomía en América Central y Suramérica, concentrados en Mexico, Venezuela, Brasil y Argentina, y solo una de ellos, Lidia Ferraro (Corrientes, Argentina), se ocupa en detalle de los líquenes foliícolas. Para muchos recolectores extranjeros no hace sentido depositar material en un herbario donde nadie lo usa. Por otrolado, para taxónomos locales es dificil empezar estudios de un grupo particular si no tienen material para la comparación. Otro obstáculo, especialmente con respecto al depósito de material tipo, es que la situación económica de herbarios tropicales a veces no garantiza el prestamo regular de especímenes.

Si queremos mejorar esta situación, tenemos que considerar varios aspectos. Primero hay que compensar el déficit de taxónomos locales.
Muchas veces es la falta de material comparativo y de literatura lo que podría retener estudiantes locales para empezar con la taxonomía, pero aún más importante es la mala perspectiva económica. En muchos países no hay fondos para financiar este tipo de estudios, y también falta la aceptación social. Es necesario que países tropicales inviertan más en investigaciones básicas, y es también necesario aplicar más continuidad en programas museísticos. No es justo que los países tropicales reclaman que sus recursos naturales son explorados científicamente por el extranjero y, para evitar eso, implementan leyes inapropriados, si al mismo tiempo niegan sus propios posibilidades y no crean una situación favorable para sus propios científicos. Muchas veces, lo único que resulta con leyes más estrictos es la mala costumbre de que cientificos extranjeros realizan sus recolecciones sin obtener permisos oficiales. Por otro lado, nosotros como liquenólogos de países extranjeros debemos estar dispuestos a ayudar con el intercambio de material y la supervisión o co-supervisión de proyectos. El inventario liquénico del Neotrópico, no solamente de los líquenes foliícolas, es una gran taréa que solo podemos realizar en colaboración.

\section{Agradecimientos}

Quiero expresar mis agradecimientos a los organizadores del GLAL-2 en Mérida, Venezuela, particularmente a Vicente Marcano, por la invitación a dar esta charla. Prof. Dr. Hannes Hertel,Dr.EmmanuëlSérusiaux,Dr.HarrieSipman y Dr. Dagmar Triebel provieron informaciones valiosas y criticamente revisaron el manuscrito.

\section{Referencias}

Alava, R. 1993. Edward August Vainio's journey to Brazil in 1885 and his Lichenes Brasilienses exsiccati. Turku.

Aptroot, A. \& H. J. M. Sipman 1993a. Trichotheliaceae. En: Görts-van Rijn, A. R. A. (ed.), Flora of the Guianas. Series E: Fungi and Lichens. Koeltz Scientific Books, Königstein.

Aptroot, A. \& H. J. M. Sipman 1993b. Musaespora, a genus of pyrenocarpous lichens with ampylidia, and 
other additions to the foliicolous lichen flora of New Guinea. Lichenologist 25: 121-135.

Aptroot, A., P. Diederich, E. Sérusiaux \& H. J. M. Sipman 1997. New or interesting lichens and lichenicolous fungi from Papua New Guinea. Bibl. Lichenol. (en prensa).

Arvidsson, L. 1986. The lichen flora of Ecuador. En Øllgaard, B. \& U. Molau (eds), Reports from the Botanical Institute, University of Aarhus, No. 15. Current Scandinavian Botanical Research in Ecuador, 13-19.

Baker, H. G. 1973. Evolutionary relationships between flowering plants and animals in American and African tropical forests. En: Meggers, B. J., E. S. Ayensu \& W. D. Duckworth (eds), Tropical forest ecosystems in Africa and South America: a comparative review, 145-159. Smithsonian Institution Press, Washington D. C.

Barillas, R. \& R. Lücking 1992. Líquenes foliícolas de Guatemala. Un estudio taxonómico preliminar. Cryptogamie, Bryol. Lichénol. 13: 297-317.

Barillas, R., R. Lücking \& S. Winkler 1993. Vergesellschaftungen foliikoler Flechten im Biotopo del Quetzal, Guatemala. Cryptogamie, Bryol. Lichénol. 14: 49-68.

Batista, A. C. 1961. Um pugilo de gêneros novos de liquens imperfeitos. Publções Inst. Micol. Recife 320: 131.

Batista, A. C. \& J. L. Bezerra 161. Arthrobotryomyces, Dothiomyces e Kilikiostroma, novos gêneros de liquens imperfeitos. Publções Inst. Micol. Recife 321: 1-20.

Batista, A. C. \& W. A. Cavalcanti 1964. Uma nova espécia de Strigula do Amazonas. Anais XIII Congr. Soc. Bot. Brasil: 472-473.

Batista, A. C. \& H. S. Maia 1961. Asbolisiomyces, Cyrta $e$ Chaetomonodorus - novos gêneros de liquens imperfeitos. Publções Inst. Micol. Recife 322: 119.

Batista, A. C. \& H. S. Maia 1965a. Algunos novos gêneros de liquens imperfeitos assinalados no IMUR. Atas Inst. Micol. 2: 351-373.

Batista, A. C. \& H. S. Maia 1965b. Caprettia, novo gênero de ascoliquen, em homenagem ao micólogo venezuelano, Corrado Capretti. Atas Inst. Micol. 2: 375-382.

Batista, A. C. \& H. S. Maia 1967. Novos liquens imperfeitos do Amazonas e de Pernambuco. Atas Inst. Micol. 5: 55-71.

Batista, A. C. \& G.E. P. Peres 1964. Liquens imperfeitos: novos gêneros e especies de Manaus. Anais XIV Congr. Soc. Bot. Brasil: 89-102

Batista, A. C. \&, D. J. M. Poroca 1970. Uma nova espécie de Echinoplaca da Amazônia. Publções Inst. Micol. Recife 635: 1-8.

Batista, A. C., J. A. Lima \& M. A. Taltasse 1962. Pycnociliospora - Um novo gênero de liquens imperfeitos. Publções Inst. Micol. Recife 251: 124.

Batista, A. C., H. S. Maia, W. F. Santos \& J. L. Bezerra 1967. Algumas especies de Mazosia comuns em o norte e nordeste do Brasil. Atas Inst. Micol. 5: 429-445.

Batista, A. C., R. C. Valle, W. A. Cavalcanti, G. E. P. Peres \& J. L. Bezerra 1961. Três novos gêneros de liquens imperfeitos, do Amazonas. Inst. Micol. Recife Inst. Nac. Pesqu. Amaz. Publica̧ao 319: 1-42.

Becker, U. \& R. Lücking 1995. Foliikole Flechten von der Elfenbeinküste, West-Afrika. I. Neue Arten. En: Daniels, F. J. A., M. Schulz \& J. Peine (eds), Contributions to lichenology in honour of Gerhard Follmann, 161-173. Geobotanical and Phytotaxonomical Study Gioup, Botanical Institute, Univ. Cologne.

Bezerra, J. L., L. Xavier Filho \& W. A. Cavalcanti 1970. Algumas especies de Trichothelium da região Amazônica. Brotéria 39: 221-229.

Bezerra, J. L., A. C. Batista, D. J. M. Poroca, W. A. Cavalcanti \& W. F. Santos 1967. Liquens e algas foliicolas de essências florestas do noroeste do Maranhão. Atas Inst. Micol. 5: 375-422.

Burger, W. C. 1980. Why are there so many kinds of flowering plants in Costa Rica? Brenesia 17: 371-388.

Cavalcante, W. A., D. J. M. Poroca, G. E. P. Peres \& F. B. Leal 1971. Contribucão ao estudo dos deuteroliquens foliicolas. Publções Inst. Micol. Recife 668: 1-17.

Cavalcante, W. A, A. A. S. A. S. Cavalcanti \& F. B. Leal 1972a. Coletãnea de liquens imperfeitos. Pulbções Inst. Micol. Recife 647: 1-46.

Cavalcante, W. A., J. L. Bezerra \& F. B. Leal 1972b. Novos ascolíquens do Brasil. Publções Inst. Micol. Recife 675: 1-17.

Coley, P. D., T. A. Kursar \& J.-L. Machado. 1993. Colonization of tropical rain forest leaves by epiphylls: effects of site and host plant leaf lifetime. Ecology 74: 619-623.

Delevoryas, T. 1973. Postdrifting mesozoic floral evo- 
lution. En: Meggers, B. J., E. S. Ayensu \& W. D. Duckworth (eds), Tropical forest eosystems in Africa and South America: a comparative review, 9-19. Smithsonian Institution Press, Washington D. C.

Dietz, R. S. \& J. C. Holden 1970. Reconstruction of Pangea: breakup and dispersion of continents. Journal of Geophysiological Research 75: 49394956.

Elix, J. A., C. E. Crook \& H. T. Lumbsch. 1992. The chemistry of foliicolous lichens. 1. Constituents of Sporopodium vezdeanum and $\mathbf{S}$. xantholeucum. Mycotaxon 44: 409-415.

Elix, J. A., H. T. Lumbsch \& R. Lücking 1995. The chemistry of foliicolous lichens 2. Constituents of some Byssoloma and Sporopodium species. Bibl. Lichenol. 58: 81-96.

Eriksson, O. 1981. The families of bitunicate ascomycetes. Opera Bot. 60: 1-220.

Farkas, E. \& H. J. M. Sipman 1993. Bibiography and checklist of foliicolous lichenized fungi up to 1992. Tropical Bryology 7: 93-148.

Farkas, E. \& A. Vezda 1993. Five new foliicolous lichn species. Folia Geobot. Phytotax., Praha, 28: 321-330.

Fée, A. L. A. 1824. Essai sur les cryptogames des écorcees exotiques officinales. Paris.

Ferraro, L. I. 1982. Contribución al estudio de los líquenes foliícolas de Corrientes, Rep. Argentina. Facena, Corrientes Argentina 4: 89-98.

Ferraro, L. I. 1983. Novedades en líquenes foliícolas. Bonplandia 5: 191-202.

Ferraro, L. I. 1990. Contribución al cononimientode los líquenes de Paraguay, colecciones de A. Schinini y de E. Bordas. Candollea 45: 655-670.

Ferraro, L. I. 1996. Checklist of foliicolous lichenized fungi from north-eastern Argentina (Corrientes and Misiones), with notes on adjacent areas of Paraguay and Brazil. En: Farkas, E. É \& Pócs, T. (eds), Cryptogams in the Phyllosphere: Systematics, Distribution, Ecology, and Use. Eszterházy Teachers' College, Eger (en prensa).

Ferraro, L. I. \& R. Lücking. 1997. New species or interesting records of foliicolous lichens. III. Arthonia crystallifera (Lichenized Ascomycetes: Arthoniaceae), with a world-wide key to the foliicolous Arthoniaceae. Phyton, Horn (en prensa).

Ferraro, L. I. \& A. Vezda 1989. Tricharia cuneata Ferraro \& Vezda sp. nov., líquen foliícola del NE de Argentina. Bonplandia 6: 111-115.

Feuerer, T. 1995. Bericht von der Tagung südamerikanischer Lichenologen. Akt. Lichenol. Mitt. Bryol.Lichenol. Arbeitsgemeinschaft Mitteleuropa 12: 5-10.

Follmann, G. \& M. Mahu 1968. Beobachtungen zur Verbreitung chilenischer Flechten. II. Strigula elegans (Fée) Müll. Arg. var. stellata (Nyl. et Cromb.) Sant. Rev. Bryol. Lichenól. 36: 333 335

Fries, E. M. 1823. Systema mycologicum II. Greifswald.

Fries, E. M. 1830. Systema mycologicum III. Greifswald.

Galloway, D. J. 1992. Lichens of Laguna San Rafael, Parque Nacional 'Laguna San Rafael', sathern Chile: indicators of environmental change. Global Ecology and Biogeography Letters 2: 37-45.

Gentry, A. H. 1982a. Neotropical floristic diversity: phytogeographical connections between Central and South America, Pleistocene climatic fluctuations, or an accident of the Andean orogeny? Ann. Missouri Bot. Gard. 69: 557-593.

Gentry, A. H. 1982b. Phytogeograplic patterns as evidence for a Chocó refuge. In Prance, G. T. (ed.). Biological diversification in the tropics, 112-136. Plenum Press, New York.

Gentry, A. H. 1986. Species richness and floristic composition of Chocó region plant communities. Caldasia 15: 71-91.

Gentry, A. H. 1988. Changes in plant community diversity and floristic composition on environmental and geographical gradients. Ann. Missouri Bot. Gard. 75: 1-34.

Gentry, A. H. \& C. H. Dodson 1987. Diversity and biogeography of neotropical vascular epiphytes. Ann. Missouri Bot. Gard. 74: 205-233.

Gottsberger, G. \& J. Döring 1995. 'COPAS', an innovative technology for long-term studies of tropical rain forest canopies. Phyton, Horn, 35: 165-173.

Gottsberger, G., E. Freiberg, A. Lücking, M. Freiberg, R. Lücking \& J. Döring 1995. Baumkronen tropischer Wälder - Interdisziplinäre Ansätze zum Verständnis der Diversität und Funktion eines wenig erforschten Lebensraums. Ulmensien 10 51-96.

Gradstein, S. R. 1987. The Ptychanthoideae of Latin America: an overview (Studies on Lejeuneaceae subfamily Ptychanthoideae XVI). Bryologist 90: 337-343.

Gradstein, S. R. 1994. Lejeuneaceae: Ptychantheae, Bra- 
chilejeuneae. Flora Neotropica Monograph 62, New York Botanical Garden, New York.

Gradstein, S. R. \& T. Pócs 1989. Bryophytes. En: Lieth, H. \& M. J. A. Werger (eds), Tropical rain forest ecosystems, 311-325. Hsevier, Amsterdam.

Grolle, R. 1968. Grossdisjunktionen in Artarealen lateinamerikanischer Lebermoose. En: Fittkau, E. J., Illies, J., Klinge, H., Schwabe, G. H. \& Sioli, H. (eds), Biogeography and ecology in South America, vol. 2, 562-582. Dr. W. Junk Publishers, The Hague.

Hafellner, J. 1984. Studien in Richtung einer natürlicheren Gliederung der Sammelfamilien Lecanoraceae und Lecideaceae. Beih. Nova Hedwigia 79: 241-371.

Harris, R. C. 1995. More Florida Lichens. The New York Botanical Garden, Bronx, New York.

Hartmann, C. 1993. Ökologsche Untersuchungen an foliikolen Flechten in Costa Rica, Zentralamerika Trabajo de diplomado, Facultad de Ciencias Naturales, Univ. Ulm.

Hartmann, C. 1996. Two new foliicolous species of Calenia (Lichens, Gomphillaceae) from Costa Rica. Mycotaxon 59: 483-488.

Henssen, A., G. Vobis \& B. Renner. 1983. New species of Rocellinastrum with an emendation of the genus. Nord. J. Bot. 2: 587-599.

Hertel. H. 1974. Krustenflechten aus Venezuela. Mitt. Bot. Staatss. München 11: 405-430.

Hertel, H. \& A. Schreiber. 1988. Die botanische Saatssammlung München 1813-1988. Mitt. Bot. Staatss. München 26: 81-512.

Kalb, K. \& A. Vezda 1988a. Die Flechtengattung Mazosia in der Neotropis (eine taxonomisch-phytogeographische Studie). Folia Geobot. Phytotax, Praha 23: 199-210.

Kalb, K. \& A. Vezda 1988b. Neue oder bemerkenswerte Arten der Flechtenfamilie Gomphillaceae in der Neotropis. Bibl. Lichenol. 29. 1-80.

Kalb, K. \& A. Vezda 1990. Die Flechtengattung Byssoloma in der Neotropis (eine taxonomisch-phytogeographische Studie). Nova Hedwigia 51: 435-451.

Kalb, K. \& A. Vezda 1992. Neue foliicole Flechten I. Nova Hedwigia 55: 195-209.

Kalb, K. \& A. Vezda 1994. Beiträge zur Kenntnis der foliicolen Flechten australischer Regenwälder IV. Bull. Soc. Linn. Provence 45: 235-246.

Krempelhuber, A. von 1876. Lichenes Brasilienses Flora 59: 1-91.

Lücking, A. 1995. Diversität und Mikrohabitatpräferenzen epiphyller Moose in einem tropischen Regenwald in Costa Rica. Dissertación, Facultad de Ciencias Naturales, Univ. Ulm. Pflanzengeografie blattbewohnender Flechten aus Costa Rica, Zentralamerika. Trabajo de diplomado, Facultad de Ciencias Naturales, Univ. Ulm.

Lücking, R. 1991. Neue Arten foliikoler Flechten aus Costa Rica, Zentralamerika. Nova Hedwigia 52: 267-304.

Lücking, R. 1992a. Foliicolous lichens - A contribution to the knowledge of the lichen flora of Costa Rica, Central America. Beih. Nova Hedwigia 104: 1179.

Lücking, R. 1992b. Zur Verbreitungsökologie foliikoler Flechten in Costa Rica, Zentralamerika. Teil 1. Nova Hedwigia 54: 309-353.

Lücking, R. 1992c. Zur Verbreitungsökologie foliikoler Flechten in Costa Rica, Zentralamerika. Teil 2. Herzogia 9: 181-212.

Lücking, R. 1993a. Schedae ad Lichenes Foliicoli Exsiccati, Fasc. I (no. 1-25). Facultad de Ciencias Naturales, Univ. Ulm.

Lücking, R. 1993b. Schedae ad Lichenes Foliicoli Exsiccati, Fasc. II (no. 26-50). Facultad de Ciencias Naturales, Univ. Ulm.

Lücking, R. 1994a. A new species of Microtheliopsis from Costa Rica, Central America. Mycotaxon 51: 69-73.

Lücking, R. 1994b. Foliikole Fechten und ihre Mkrohabitatpräferenzen in einem tropischen Regenwald in Costa Rica. Dissertación, Facultad de Ciencias Naturales, Univ. Ulm.

Lücking, R. 1995a Additions and corrections to the foliicolous lichen flora of Costa Rica, Central America. The family Arthoniaceae, with notes on the genus Stirtonia. Lichenologist 27: 127153.

Lücking, R. 1995b. Foliikole Flechten auf Cecropiaceen im Kronendach eines tropischen Regenwaldes. Bibl. Lichenol. 58: 261-274.

Lücking, R. 1995c. Biodiversity and conservation of foliicolous lichens in Costa Rica. Mitteil. Eidgn. Forsch.anstalt Wald, Schnee Landsh. 70: 6392.

Lücking, R. 1995d. Schedae ad Lichenes Foliicoli Exsiccati, Fasc. III (no. 51-75). Facultad de Ciencias Naturales, Univ. Ulm.

Lücking, R. 1995e. Schedae ad Lichenes Foliicoli Exsic- 
cati, Fasc. IV (no. 76-100). Facultad de Ciencias Naturales, Univ. Ulm.

Lücking, R. 1995f. Schedae ad Lichenes Foliicoli Exsiccati, Fasc. V (no. 101-125). Facultad de Ciencias Naturales, Univ. Ulm.

Lücking, R. 1995g. Schedae ad Lichenes Foliicoli Exsiccati, Fasc. VI (no. 126-150). Facultad de Ciencias Naturales, Univ. Ulm.

Lücking, R. 1996a. Taxonomic studies in foliicolous species of the genus Porina. I. The Porina rufula aggregate. Bot. Acta 109: 248-260.

Lücking, R. 1996b. Notes on "Lichenes Foliicoli Exsiccati", Fasc. I-VI. En: Farkas, E. É \& Pócs, T. (eds), Cryptogams in the Phyllosphere: Systematics, Distribution, Ecology, and Use. Eszterházy Teachers' College, Eger (en prensa).

Lücking, R. 1996c. The use of foliicolous lichens as bioindicators in the tropics, with special reference to the microclimate. En: Farkas, E. É \& Pócs, T. (eds), Cryptogams in the Phyllosphere: Systematics, Distribution, Ecology, and Use. Eszterházy Teachers' Cdlege, Eger (en prensa).

Lücking, R. 1996d. Lista preliminar de líquenes foliícolas de las principales areas protegidas de Costa Rica. Brenesia (en prensa).

Lücking, R. 1997a. Additions and corrections to the knowledge of the foliicolous lichen flora of Costa Rica, Central America. The genus Trichothelium. Nova Hedwigia (en prensa).

Lücking, R. 1997b. Additions and corrections to the knowledge of the foliicolous lichen flora of Costa Rica, Central America. The genus Fellhanera, with notes on Bacidia pauciseptata. Tropical Bryology (en prensa).

Lücking, R. 1997c. Additions and corrections to the knowledge of the foliicolous lichen flora of Costa Rica, Central America. The family Gomphillaceae. Bibl. Lichenol. (en prensa).

Lücking, R. \& L. I. Ferraro. 1997. New species or interesting records of foliicolous lichens. I. Trichothelium argenteum (Lichenized Ascomycetes: Trichotheliaceae). Lichenologist (en prensa).

Lücking, R. \& A. Lücking 1995. Foliicolous lichens and bryophytes from Cocos Island, Costa Rica. A taxonomical and ecogeographical study. I. Lichens. Herzogia 11: 143-174.

Lücking, R. \& M. Matzer 1996. Ergänzungen und Verbesserungen zur Kenntnis der foliikolen Flechtenflora von Costa Rica. Die Familie Opegraphaceae (einschließlich der Gattung
Mazosia). Nova Hedwigia 63: 109-144.

Lücking, R. \& A. Vezda 1995. Proposal to conserve Badimia against Pseudogyalecta (Lichenized Ascomycotina). Taxon 44: 227-228.

Lücking, R., T.H. Lumbsch \& J. A. Elix 1994. Chemistry, anatomy and morphology of foliicolous species of the genera Fellhanera and Badimia (lichenized ascomycotina: Lecanorales). Bot. Acta 107: 393-401.

Lücking, R., E. Sérusiaux, L. C. Maia \& E. C. G. Pereira 1997a. A taxonomic revision of names of foliicolous, lichenized fungi published by Batista and co-workers between 1960 and 1975 . Lichenologist (en prep.).

Lücking, R., U. Becker \& G. Follmann. 1997a. Foliikole Flechten aus dem Taï-Nationalpark, Elfenbeinküste (Tropisches Westafrika). II. Ökologie und Biogeographie. Herzogia (en prep.).

Lynge, B. 1915. Index speciarum et varietatum lichenorum collectionibus "Lichenes exsiccati" distributae sunt. Kristiania I + II.

Malcolm, W. M. \& A. Vezda 1994. Badimiella serusiauxii, a new genus and species of foliicolous lichens from New Zealand (Ectolechiaceae). Nova Hedwigia 59: $517-523$.

Malcolm, W. M. \& A. Vezda 1995. New foliicolous lichens from New Zealand 1. Folia Geobot. Phytotax., Praha, 30: 91-96.

Malme, G. O. 1924. Die Flechten der ersten Regnellschen Expedition. Astrotheliaceae, Paratheliaceae und Trypetheliaceae. Arkiv. Bot. 19A: 1-34.

Malme, G. O. 1928. Lichenes pyrenocapi aliquot in herbario Regnelliano asservati. Arkiv. Bot. 22A: $1-11$.

Malme, G. O. 1929. Porinae et Phylbporinae in Itinere Regnelliano primo collectae. Arkiv Bot. 23A: 137.

Malme, G. O. 1934. Die Gyalectazeen der ersten RegneH schen Expedition. Arkiv. Bot. 26A: 1-10.

Malme, G. O. 1935. Actiniopsis Starb., Stereochlamys Müll. Arg. und Trichothelium Müll. Arg. Hedwigia 75.

Malme, G. O. 1937. Lichenes nonulli in Expeditione Regnelliana prima collecti. Arkiv. Bot. 29A: 135.

Martin, H. 1968. A critical review of the evidence for a former direct connection of South America with Africa. En: Fittkau, E J., Illies, J., Klinge, H., Schwabe, G. H. \& Sioli, H. (eds), Biogeography and ecology in South America, vol. 1, 25-53. Dr. 
W. Junk Publishers, The Hague.

Montagne, C. 1838-1842. Botanique. Plantes cellulaires

En: Sagra, R. de la (ed.), Histoire de l'îsle de Cuba, vol. 9: 2. Paris.

Montagne, C. 1843. Quatrième centurie de plantes cellulaires exotiques nouvelles. Décades VIII, IX et X, 352-379. Annales des Sciences Naurelles, Botanicae, series 2, vol. 20. Paris.

Montagne, C. 1851. Cryptogamia Guyanensis. Annales Scientiarum Naturarum Botanicae, series 3, vol. 16. Paris.

Montagne, C. 1856. Sylloge generum specierumque cryptogamarum. Bibliopolae Academiae Imperialis Medicinae. J.-P. Baillière, Paris.

Montfoort, D. \& R. C. Ek 1990. Vertical distribution and ecology of epiphytic bryophytes and lichens in a tropical lowland rain forest in French Guiana. Institute of Systematic Botany, Utrecht.

Müller, P. 1973. The dispersal centres of terrestrial vertebrates in the neotropical realm. Dr. W. Junk Publishers, The Hague.

Müller Argoviensis, J. 1885. Pyrenocarpae Cubenses a cl. C. Wright lectae. Botanische Jahrbücher 6: 375 421.

Müller Argoviensis, J. 1888a. Lichenes Paraguayenses. Rev. Mycol. 38: 53-68, 113-120, 177-184.

Müller Argoviensis, J. 1888b. Pyrenocarpae Feeanae. Mem. Soc. Phys. Hist. Nat. Geneve 30: 1-45.

Müller Argoviensis, J. 1888c. Lichenes Portoricenses. Flora 71: 1-7.

Müller Argoviensis, J. 1890. Lichenes epiphylli novi. Georg \& Richter, Genève.

Müller Argoviensis, J. 1891. Lichenes. En: Durand \& H. Pittier (ed.) Primitiae florae costaricensis. Bull. Soc. Bot. Belg 30: 47-95.

Müller Argoviensis, J. 1892. Lichenes epiphylli Spruceani. Linn. Soc. Journ. Bot. 29. 322-333.

Müller Argoviensis, J. 1894a. Lichenes. Seconde Énumération. En: Durand \& H. Pittier (ed.) Primitiae florae costaricensis. Bull. Soc. Bot. Belg. 32.

Müller Argoviensis, J. 1894b. Arthoniae et Arthothelii species Wrightianae in insula Cuba lectae. Bull. Herb. Boiss. 2: 725-736.

Müller Argoviensis, J. 1895. Lichenes Uleani in Brasilia lecti. Hedwigia 34: 39-42.

Nowak, R. \& S. Winkler 1970. Foliicole Flechten der Sierra Nevada de Santa Marta (Kolımbien) und ihre gegenseitigen Beziehungen. Österr. Bot. Z. 118: 456-485.

Nowak, R. \& S. Winkler 1972. Foliicole Flechten von
El Salvador, C. A. Rev. Bryol. Lichenól. 38: 269279.

Nowak, R. \& S. Winkler 1975. Foliicolous lichens of Chocó, Colombia, and their substrate abundances. Lichenologist 7: 53-58.

Osorio, H. S. 1970a. Contribution to he lichen flora of Uruguay V. Lichens from "Paso Yacare", Salto County. Comun. Bot. Mus. Hist. Nat. Monteideo 52: 1-2.

Osorio, H. S. 1970b. Contribution to the lichen flora of Argentina V. Some new records. Comun. Bot. Mus. Hist. Nat. Montevideo 54: 1-2.

Osorio, H. S. 1972. Contribution to the lichen flora of Uruguay VII. A preliminary catalogue. Comun. Bot. Mus. Hist. Nat. Montevideo 56: 1-46.

Osorio, H. S. 1975. Contribution to the lichen flora of Uruguay VIII. Additions and corrections. Comun. Bot. Mus. Hist. Nat. Montevideo 59: 1-12.

Osorio, H. S. 1992. Contribución a la flora liquénica del Uruguay. XXV. Líquenes publicados entre 1972 a 1991. Anal. Mus. Nac. Hist. Nat. Montevideo 8: 43-70.

Prance, G. T. 1977. Floristic inventory of the tropics: where do we stand? Ann. Missouri Bot. Gard. 64: 659-684.

Prance, G. T. 1982 (ed.). Biological diversification in the tropics. Columbia University Press, New York.

Raven, P. H. \& D. Axelrod 1974. Angiosperm biogegraphy and past continental movements. Ann. Missouri Bot. Gard. 61: 539-673.

Ricci, P. \& R. Tomaselli 1958. Licheni foliicoli raccolti da E. Mameli Calvino. Archo Bot. Biogeogr. Ital. 34, ser. 4, 3: 254-262.

Richards, P. W. 1984. The ecology of ropical forest bryophytes. En: Schuster, R. M. (ed) New manual of bryology, vol. 2, 1233-1270. Hatori Botanical Laboratory, Nidinan.

Rogers, R. W., A. Barnes \& J. G. Conran 1994. Lichen succession on Wilkiea macrophylla leaves. Lichenologist 26: 135-147.

Santesson, J. 1970. Chemical studies on lichens. 28. The pigments of some foliicolous lichens. Acta Chemica Scand. 24: 371-373.

Santesson, R. 1952. Foliicolous lichens I. Symb. Bot. Ups. 12(1): 1-590.

Sayre, G. 1969. Cryptogamae exsiccatae - an annotated bibliography of published exsiccatae of algae, lichens, hepatics, and musci. Memoirs New York Bot. Gard. 19: 1-174.

Schell, S. \& S. Winkler 1980. Zur Ökologie und Pflan- 
zengeographie blattbewohnender Flechten vo Rio Grande do Sul (Südbrasilien. Cryptogamie, Bryol. Lichenól. 2: 323-343.

Schilling, F. 1927. Entwicklungsgeschichtliche und systematische Untersuchung epiphyller Hechten. Hedwigia 67.

Sérusiaux, E. 1976. Some foliicolous lichens from the Farlow Herbarium I. Occ. Pap. Farlow Herb. 10 1-21.

Sérusiaux, E. 1977. Quelques lichens foliicdes récoltés à La Réunion (Afrique, Océan Indien). Bull. Soc. Roy. Bot. Belg. 110: 39-41.

Sérusiaux, E. 1978. Contribution à l'étude de lichens du Kivu (Zaïre), du Rwanda et du Burundi II. Espèces nouvelles de lichens foliicdes. Lejeunea N. S. 90: $1-18$.

Sérusiaux, E. 1979a. Contribution to the study of lichens from Kivu (Zaïre), Rwanda and Burundi III. Vez daea, a new genus for Africa. Mycotaxon 8: 135-139.

Sérusiaux, E. 1979b. Two new foliicolous lichens from tropical Africa. Lichenologist 11: 181-185.

Sérusiaux, E. 1983. Foliicolous lichens from Zimbabwe. Lichenologist 15: 283-287.

Sérusiaux, E. 1984. New species o interesting records of foliicolous lichens. Mycotaxon 20: 283-306.

Sérusiaux, E. 1985. Goniocysts, goniocystangia and Opegrapha lambinonii and related species. Lichenologist 17: 1-25.

Sérusiaux, E. 1986. The nature and origin of campylidia in lichenized fungi. Lichenologist 18: 1-35.

Sérusiaux, E. 1989. Foliicolous lichens: ecobgical and chorological data. Bot. J. Linn. Soc. 100: 87-96.

Sérusiaux, E. 1992. Reinstatement of the lichenized genus Eremothecella Syd. Systema Ascomycetum 11: 39-47.

Sérusiaux, E. 1993. New taxa of foliicolous lichens frm Western Europe and Macaronesia. Nord. J. Bot. 13: 447-461.

Sérusiaux, E. 1995. Further new lichen specis with campylidia or complex conidiomata. Bibl. Lichenol. 58: 411-431.

Sérusiaux, E. \& J. R. de Sloover 986. Taxonomical and ecological observations on foliicolous lichens in northern Argentina, with notes on the hyphophores of Asterothyriaceae. Veröff. Geobot. Inst. ETH, Stiftung Rübel, Zürich 91: 260-292.

Sick, W.-D. 1968. Geographical substance. En: Fittkau, E. J., Illies, J., Klinge, H., Schwabe, G. H. \& Sioli,
H. (eds), Biogeography and ecology in South America, vol. 2, 449-474. Dr. W. Junk Publishers, The Hague.

Sipman, H. J. M. 1990a. Colección preliminar de líqenes sobre hojas en Araracuara, Colombia. Colombia Amazonica 4: 59-65.

Sipman, H. J. M. 1990b. Lichenotheca Latinoamericana a museo botanico berolinensi edita, fasciculum primum. Willdenowia 19: 543-551.

Sipman, H. J. M. 1991a. Notes on the lichen flora of the Guianas, a neotropical lowland area. En: Galloway, D. J. (ed.), Tropical Lichens: their systematics, conservation, and ecology. Systematics Association Special Volume 43: 135-150. Clarendon Press, Oxford.

Sipman, H. J. M. 1991b. Observations on foliicolous lichens in the Guianas. Flora of the Guianas Newsletter 8: 8-9.

Sipman, H. J. M. 1992. Results of a lichenological and bryological exploration of Cerro Guaiquinima (Guyana Highland, Venezuela). Tropical Bryology 6: 1-31.

Sipman, H. J. M. 1993a. Lichens from Mount Kinabalu. Tropical Bryology 8: 281-314.

Sipman, H. J. M. 1993b. Lichenotheca Latinoamericam a museo botanico berolinensi edita, fasciculum secundum. Willdenowia 23: 305-314.

Sipman, H. J. M. 1996. Observations on the foliiolous lichen and bryophyte flora in the canopy of a semi-deciduous tropical forest. En: Farkas, E. É \& Pócs, T. (eds), Cryptogams in the Phyllosphere: Systematics, Distribution, Ecology, and Use. Eszterházy Teachers' College, Eger (en prensa).

Sipman, H. J. M. \& A. Aptroot 1992. Results of a botanical expedition to Mount Roraima, Guyana. II. Lichens. Tropical Bryology 5: 79-107.

Sipman, H. J. M. \& R. C. Harris 1989. Lichens. En: Lieth, H. \& M. J. A. Werger (eds), Tropical rain forest ecosystems, 303-309. Elsevier, Amsterdam.

Smith, A. V. 1973. Angiosperm evolution and relationships of the floras of Africa and America. En: Meggers, B.J., Ayensu, E.S. \& Duckworth, W.D. (eds), Tropical forest ecosystems in Africa and South America: A comparative review, 4961. Smithsonian Institution Press, Washington, D.C

Sørensen, T. 1948. A method of establishing groups of equal amplitude in plant sociology based on similarity of species content. Biologiske Skrifter K. Danske Videnskaberness Selskab 5: 1-34. 
Stirton, J. 1878. Lichens growing on living leaves from the Amazonas. Proc. Philos. Soc. Glasgow 11.

Thor, G., R. Lücking \& T. Matsumoto. 1997. The foliicolous lichen flora of Japan. Opera Botanica (en prep.).

Thorne, R. F. 1973. Floristic relationships between tropical Africa and tropical America. En: Meggers, B.J., Ayensu, E.S. \& Duckworth, W.D. (eds), Tropical forest ecosystems in Africa and South America: A comparative review, 2747. Smithsonian Institution Press, Wahington, D.C.

Tibell, L. 1994. Distribution patterns and dispersal strategies of Caliciales. Bot. J. Linn. Soc. 116: 159202 .

Upadhyay, H. B. P. 1964a. Three new hyperparasites for Mazosia phyllosema (Nyl.) A. Zahlbr. from Amazonas valley. Publções Inst. Micol. Recife 402: 111.

Upadhyay, H. B. P. 1964b. A new Opegrapha species from the Federal Territóry of Rondônia. Publções Inst. Micol. Recife 410: 1-6.

Vainio, E. A. 1890. Étude sur la classfication naturelle et la morphologie des lichens du Brésil. I-II. Acta Soc. Fauna Fl. Fennica 7.

Vainio, E. A. 1896. Lichenes antillarum $a W$. R. Elliott collecti. Journal of Botany 34

Vareschi, V. 1953. La influencia de los bosqus y parques sobre el aire de la ciudad de Caracas. Acta Cienc. Venez. 4: 89-95.

Vareschi, V. 1973. Catálogo de los líquenes de Venezued. Acta Bot. Venez. 8: 177-245.

Vezda, A. 1973. Foliicole Flechten aus der Republik Guinea (W-Afrika). I. Acta Mus. Sil., Opava, ser. A 22: 67-90.

Vezda, A. 1974. Foliicole Flechten aus der Republik Guinea (W-Afrika). II. Acta Mus. Sil., Opava, ser. A 23: 173-190.

Vezda, A. 1975a. Foliicole Flechten aus der Republik Guinea (W-Afrika). III. Acta Mus. Sil., Opava, ser. A 24: 117-126.

Vezda, A. 1975b. Foliikole Flechten aus Tanzania (OstAfrika). Folia Geobot. Phytotax., Praha, 10 383-432.

Vezda, A. 1979. Flechtensystematische Sudien XI. Beiträge zur Kenntnis der Familie Asterothyriaceae (Discolichenes). Folia Geobot. Phytotax., Praha, 14: 43-94

Vezda, A. 1980. Foliikole Flechten aus Zaïre. Die Arten der Sammelgattungen Catillaria und Bacidia. Folia
Geobot. Phytotax., Praha, 15: 75-94.

Vezda, A. 1982. Foliikole Flechten aus Zaïre. (II). Zwei neue Arten der Gattung Pocsia. Folia Geobot. Phytotax., Praha, 17: 387-392.

Vezda, A. 1984. Foliikole Flechten der Insel Kuba. Folia Geobot. Phytotax., Praha, 19: 177-210.

Vezda, A. 1986. Neue Gattungen der Famile Lecideaceae s. lat. (Lichenes). Folia Geobot. Phytotax., Praha, 21: $199-219$.

Vezda, A. 1987. Foliikole Flechten aus Zaïre. (III). Die Gattung Byssoloma Trevisan. Folia Geobot. Phytotax., Praha, 22: 71-83.

Vezda, A. 1990. Bacidina genus novum familiae Lecideaceae s. lat. (Ascomycetes lichenisati). Folia Geobot. Phytotax., Praha, 25: 431-432

Vezda, A. 1994. Neue foliicole Flechten II. Nova Hedwigia 58: $123-143$

Vezda, A. \& E. Farkas. 1988. Neue foliicole Arten der Flechtengattung Dimerella Trevisan (Gyalectaceae) aus Tansania. Folia Geobot. Phytotax., Praha, 23: 187-197.

Vezda, A. \& R. Lücking 1995. A estudy of Pseudogyalecta verrucosa, its systematical affinities, and the nomenclatorical consequences. Mycotaxon 55: 501-506.

Vobis, G. \& D. L. Hawksworth. 1981. Conidial lichen.forming fungi. En: Cole, G. T. \& B. Kendrick (eds), Biology of conidial fungi, 245-273. Academic Press, New York, London

Wolseley, P. A. 1991. Observations on the composition and distribution of the 'Lobarion' in foests of South East Asia. En: Galloway, D. J (ed), Tropical lichens: their systematics, conservation, and ecology. Systematics Association Special Vol. 43, 217-243. Clarendon Press, Oxford.

Wolseley, P. A. \& B. Aguirre-Hudson, B. 1991. Lichens as indicators of environmental change in the tropical forests of Thailand. Global Ecology and Biogeography Letters 1: 170-175.

Wolseley, P. A., C. Moncrieff \& B. Aguirre-Hudson. 1994. Lichens as indicators of environmental stability and change in the trqical forest of Thailand. Global Ecology and Biogeography Letters 4: 116-123.

Xavier Filho, L. 1973. Um novo Arthonia e outros líquens estudados no IMUR. Publções Inst. Micol. Recife 357: 1-16.

Xavier Filho, L. 1975. Raciborskiella parva Xavier Filho, nova espécie de Strigulaceae (Líquens) do Estado do Amazonas. Acta Amazonica 5: 141-142. 
Xavier Filho, L. 1976a. Phragmopeltecaceae uma nova família de microascolíquens. Universidade Federal da Paraíba, Centro de Ciências Exatas e da Natureza, João Pessôa, Paraíba, 1-80.

Xavier Filho, L. 1976b. Lopadium couepiae Xavier Filho nova espécie de Lecideaceae (Liquen) do Estado de Amazonas. Anais da UfRPE, Ciencias Biológicas Recife 3: 95-98.

Xavier Filho, L. \& L. M. Barros 1970. Revisão da coleção de liquens do Museo Goeldi. Univ. Federal de Pernambuco. Inst. Biodencias, Ser. B, 1: 1-7.

Zahlbruckner, A. 1908. Beiträge zur Flechtenflora Brasiliens. Bull. Herb. Boiss., ser. 2, vol. 8: 459-468.

Zahlbruckner, A. 1909. Lichenes (Flechten). En: Ergebnisse der Botanischen Expedition der Kaiserlichen Akademie der Wissenschaften nach Südbrasilien 1901. Denkschrift Math.-Nat. Kl. Akad. Wien 83: 87-211 + 5 Tafeln.

Zahlbruckner, A. 1930. New species of lichens from Puerto Rico. III. Myølogia 22. 